\title{
35. DATA REPORT: SUMMARY OF GEOCHEMICAL DATA FOR LEG 129 IGNEOUS ROCKS ${ }^{1}$
}

\author{
Paterno R. Castillo, ${ }^{2}$ Peter A. Floyd,${ }^{3}$ Christian France-Lanord, ${ }^{4}$ and Jeffrey C. Alt ${ }^{5}$
}

This report presents all the available major and trace elemental analyses and $\mathrm{Sr}, \mathrm{Nd}$, and $\mathrm{Pb}$ isotopic compositions of basaltic rocks recovered from Ocean Drilling Program Sites 800, 801, and 802 during Leg 129 (Table 1). Its main purpose is to provide other investigators a complete summary of geochemical data for Leg 129 basement basalts that they can use for later work. Detailed discussions of the data are presented elsewhere in the volume by Floyd and Castillo (Site 801 geochemistry and petrogenesis), Floyd et al. (Sites 800 and 802 geochemistry and petrography), Alt et al. (Site 801 alteration), and Castillo et al. ( $\mathrm{Sr}, \mathrm{Nd}$, and $\mathrm{Pb}$ isotope geochemistry of Leg 129 basalts).

A vast majority of the major oxide and trace elemental analyses were done by standard X-ray fluorescence (XRF) method at the Geoscience Analytical Services, University of Keele (M. Aikin and D. Emley, analysts). A few (22) of the determinations were done at the Department of Geological Sciences, The University of Michigan (labeled UM in Table 1; J. Alt, analyst). Loss on ignition (LOI) were also determined at these two laboratories. Major oxides were analyzed in fused glass beads and trace elements $\mathrm{Ba}, \mathrm{Co}, \mathrm{Cr}, \mathrm{Cu}, \mathrm{Ga}, \mathrm{Nb}, \mathrm{Ni}$, $\mathrm{Pb}, \mathrm{Rb}, \mathrm{S}, \mathrm{Sr}, \mathrm{V}, \mathrm{Y}, \mathrm{Zn}$, and $\mathrm{Zr}$ in pressed powder pellets. $\mathrm{FeO}$ was determined by titration with potassium dichromate. Based on repeated analyses of rock standards, the analytical precision (measured by the coefficient of variation) for major oxides is generally better than $1.5 \%$ and for trace elements is better than $5 \%$.

Contents of $\mathrm{H}_{2} \mathrm{O}^{+}$and $\mathrm{CO}_{2}$ of aliquots of powders analyzed for major and trace elements were determined at the Centre de Recherches Petrographiques et Geochimiques (M. Vernet, analyst). Water was determined by volumetry. Samples were preheated at $105^{\circ} \mathrm{C}$, weighed and

'Larson, R. L., Lancelot, Y., et al., 1992. Proc. ODP, Sci. Results, 129: College Station, TX (Ocean Drilling Program).

${ }^{2}$ GRD, Scripps Institution of Oceanography, La Jolla, CA 92093-0220, U.S.A.

${ }^{3}$ Department of Geology, University of Keele, Staffordshire, ST5 5BG, United Kingdom.

${ }^{4}$ Centre de Recherches Petrographiques et Geochimiques, F-54501 Vandoeuvre, Cedex, France.

${ }^{5}$ Department of Geological Sciences. The University of Michigan, Ann Arbor, MI 48109-1063, U.S.A. then heated again at $1400^{\circ} \mathrm{C}$ in a tubular furnace. Released water was measured by titration using the dynamic Karl Fisher method. Standard deviation is 0.017 at $0.5 \mathrm{wt} \%$ and 0.045 at $3 \mathrm{wt} \%$. Carbon dioxide from carbonate analyses were done using a Beckman DU-62 coulometer. Standard deviation is 0.01 at $0.1 \mathrm{wt} \%$ and 0.02 at $1 \mathrm{wt} \%$.

Other trace elements such as $\mathrm{Co}, \mathrm{Cs}, \mathrm{Hf}, \mathrm{Sc}, \mathrm{Ta}, \mathrm{Th}, \mathrm{U}$, and the rare earths ( $\mathrm{La}$ to $\mathrm{Lu})$ were determined in the same powders of a subset of the samples analyzed by XRF at the University of Keele by instrumental neutron activation analysis (INAA) at the Department of Earth and Planetary Sciences, Washington University (labeled WU in Table 1; R. Korotev, analyst) and Universities Research Reactor, Risley (G. Gilmore, analyst). The rare earth element contents of a few samples were analyzed by inductively coupled plasma (ICP) emission spectrometry at Royal Holloway and Bedford New College, University of London (N. Walsh, analyst) and ICP results are basically identical to the Universities Research Reactor INAA results (Floyd and Castillo, this volume); these two types of trace element analyses were all reported as INAA data in Table 1. In general, data for four samples collected at the two INAA facilities also show reasonable agreement (Table 2).

Isotopic composition measurements were done on leached powders of samples from Sites 800 and 801 to correct for seawater alteration; those for Site 802 samples were done on hand-picked basaltic glasses. All $\mathrm{Pb}$ isotopic and most $\mathrm{Sr}$ and $\mathrm{Nd}$ isotopic analyses were done by thermal ionization mass spectrometry at the Department of Terrestrial Magnetism, Carnegie Institution of Washington (P. Castillo, analyst). A few $\mathrm{Sr}$ and $\mathrm{Nd}$ isotopic measurements were done at the Geological Survey Division, Scripps Institution of Oceanography. Analytical precision for ${ }^{206} \mathrm{~Pb} /{ }^{204} \mathrm{~Pb}$ is \pm 0.015 , ${ }^{207} \mathrm{~Pb} / 204 \mathrm{~Pb}$ is $\pm 0.019,{ }^{208} \mathrm{~Pb} /{ }^{204} \mathrm{~Pb}$ is $\pm 0.060,{ }^{87} \mathrm{Sr} /{ }^{86} \mathrm{Sr}$ is \pm 0.000020 , and ${ }^{143} \mathrm{Nd} /{ }^{144} \mathrm{Nd}$ is \pm 0.000023 ( \pm 0.000017 at Scripps).

Date of initial receipt: 13 June 1991

Date of acceptance: 4 December 1991

Ms 129B-145 
Table 1. Major and trace element and isotopic composition of Leg 129 igneous rocks.

\begin{tabular}{|c|c|c|c|c|c|c|c|c|c|}
\hline Hole & $800 \mathrm{~A}$ & $800 \mathrm{~A}$ & $800 \mathrm{~A}$ & $800 \mathrm{~A}$ & $800 \mathrm{~A}$ & $800 \mathrm{~A}$ & $800 \mathrm{~A}$ & $800 \mathrm{~A}$ & $800 \mathrm{~A}$ \\
\hline Core-Sec & $57 R-1$ & $57 \mathrm{R}-1$ & $57 R-2$ & $57 R-2$ & $58 \mathrm{R}-1$ & $58 \mathrm{R}-1$ & $58 \mathrm{R}-1$ & $58 \mathrm{R}-1$ & $58 \mathrm{R}-2$ \\
\hline Top-Bottom & $17-24$ & $40--45$ & $49-.53$ & $86--91$ & $12--17$ & $47--51$ & $86--91$ & $127--131$ & $32-37$ \\
\hline Depth (mbsf) & 498.17 & 498.4 & 498.99 & 499.36 & 507.32 & 507.67 & 508.06 & 508.47 & 508.85 \\
\hline Cooling Unit & 1 & 1 & 1 & 1 & 2 & 3 & 3 & 3 & 3 \\
\hline Major oxides by XRF (wt. \%) & & & UM & & & & & UM & \\
\hline $\mathrm{SiO}_{2}$ & 49.56 & 45.72 & 43.30 & 49.09 & 49.34 & 49.88 & 50.60 & 48.60 & $\overline{48.94}$ \\
\hline $\mathrm{TiO}_{2}$ & 2.17 & 1.95 & 1.80 & 2.01 & 2.19 & 2.21 & 2.13 & 2.06 & 2.11 \\
\hline $\mathrm{Al}_{2} \mathrm{O}_{3}$ & 15.86 & 14.77 & 14.50 & 15.97 & 15.91 & 15.69 & 15.89 & 15.40 & 15.83 \\
\hline $\mathrm{Fe}_{2} \mathrm{O}_{3}$ & 9.01 & 6.84 & 7.00 & 5.63 & 4.51 & 5.55 & 4.58 & 4.65 & 5.72 \\
\hline $\mathrm{FeO}$ & 0.37 & 1.06 & 1.50 & 2.82 & 4.92 & 4.01 & 5.12 & 4.90 & 3.78 \\
\hline $\mathrm{MnO}$ & 0.15 & 0.12 & 0.12 & 0.07 & 0.15 & 0.15 & 0.19 & 0.23 & 0.20 \\
\hline $\mathrm{MgO}$ & 5.04 & 4.01 & 5.03 & 7.92 & 6.72 & 6.76 & 6.44 & 6.21 & 6.46 \\
\hline $\mathrm{CaO}$ & 3.96 & 10.27 & 11.30 & 4.83 & 6.00 & 4.53 & 5.92 & 6.02 & 6.34 \\
\hline $\mathrm{Na}_{2} \mathrm{O}$ & 2.66 & 3.63 & 2.92 & 2.94 & 3.92 & 3.86 & 4.12 & 3.65 & 4.07 \\
\hline $\mathrm{K}_{2} \mathrm{O}$ & 5.64 & 3.69 & 2.62 & 3.22 & 2.55 & 2.88 & 2.28 & 2.26 & 1.97 \\
\hline $\mathrm{P}_{2} \mathrm{O}_{5}$ & 0.55 & 0.55 & 0.50 & 0.56 & 0.54 & 0.62 & 0.54 & 0.55 & 0.55 \\
\hline LOI & 4.94 & 8.04 & 9.08 & 5.30 & 3.49 & 3.21 & 2.63 & 4.31 & 3.50 \\
\hline Total & 99.9 & 100.7 & 99.8 & 100.4 & 100.2 & 99.4 & 100.4 & 99.4 & 99.5 \\
\hline $\mathrm{CO}_{2}$ tot & 1.34 & 5.10 & 5.53 & 1.38 & 1.18 & 0.22 & 0.60 & 0.62 & 0.64 \\
\hline $\mathrm{H}_{2} \mathrm{O}^{+}$ & 3.38 & 2.21 & 2.50 & 3.61 & 2.38 & 3.04 & 2.13 & 2.30 & 2.50 \\
\hline Trace element by XRF (ppm) & & & UM & & & & & UM & \\
\hline $\mathrm{Ba}$ & 475 & 251 & 267 & 339 & 337 & 388 & 317 & 383 & 368 \\
\hline Co & - & - & 33 & - & - & - & - & 33 & - \\
\hline $\mathrm{Cr}$ & 203 & 140 & 110 & 127 & 134 & 80 & 155 & 81 & 81 \\
\hline $\mathrm{Cu}$ & 63 & 30 & 48 & 65 & 54 & 24 & 36 & 73 & 83 \\
\hline $\mathrm{Ga}$ & 21 & 22 & - & 23 & 22 & 22 & 22 & - & 24 \\
\hline $\mathrm{Nb}$ & 52 & 48 & 47 & 50 & 51 & 54 & 53 & 55 & 53 \\
\hline $\mathrm{Ni}$ & 122 & 83 & 73 & 82 & 42 & 43 & 41 & 40 & 46 \\
\hline $\mathrm{Pb}$ & 3 & 4 & - & 1 & 1 & 1 & 2 & - & 2 \\
\hline $\mathrm{Rb}$ & 93 & 82 & 90 & 20 & 31 & 22 & 19 & 32 & 24 \\
\hline S & 123 & 128 & - & 1054 & 273 & 263 & 332 & - & 421 \\
\hline $\mathrm{Sr}$ & 340 & 466 & 436 & 493 & 566 & 525 & 608 & 255 & 578 \\
\hline V & 165 & 188 & - & 185 & 187 & 183 & 186 & - & 192 \\
\hline $\mathrm{Y}$ & 22 & 25 & 16 & 23 & 27 & 27 & 26 & 31 & 25 \\
\hline $\mathrm{Zn}$ & 137 & 80 & 88 & 144 & 77 & 101 & 72 & 110 & 117 \\
\hline $\mathrm{Zx}$ & 348 & 330 & 222 & 340 & 356 & 360 & 390 & 249 & 362 \\
\hline Trace element by INAA (ppm) & & WU & & WU & & & & & \\
\hline Co & - & 52 & - & 33 & - & - & - & - & - \\
\hline Cs & - & 3.53 & - & 0.14 & 0.15 & 0.15 & - & - & - \\
\hline $\mathrm{Hf}$ & - & 7.51 & - & 7.12 & 7.70 & 8.10 & - & - & - \\
\hline Sc & - & 16.30 & - & 17.80 & 20.30 & 18.60 & - & - & - \\
\hline $\mathrm{Ta}$ & - & 3.18 & - & 3.21 & 3.26 & 3.33 & - & - & - \\
\hline $\mathrm{Th}$ & - & 3.57 & - & 3.64 & 4.20 & 4.30 & - & - & - \\
\hline $\mathrm{U}$ & - & 1.93 & - & 1.58 & 1.25 & 1.23 & - & - & - \\
\hline $\mathrm{La}$ & - & 37.10 & - & 36.50 & 43.06 & 46.07 & - & - & - \\
\hline $\mathrm{Ce}$ & - & 79.80 & - & 78.30 & 87.32 & 93.87 & - & - & - \\
\hline $\operatorname{Pr}$ & - & - & - & - & 9.90 & 10.81 & - & - & - \\
\hline $\mathrm{Nd}$ & - & 36.00 & - & 37.00 & 40.25 & 43.77 & - & - & - \\
\hline $\mathrm{Sm}$ & - & 7.96 & - & 7.75 & 7.96 & 8.44 & - & - & - \\
\hline $\mathrm{Eu}$ & - & 2.48 & - & 2.46 & 2.57 & 2.72 & - & - & - \\
\hline Gd & - & - & - & - & 6.84 & 7.44 & - & - & - \\
\hline $\mathrm{Tb}$ & - & 0.94 & - & 0.87 & - & - & - & - & - \\
\hline Dy & - & - & - & - & 5.33 & 5.55 & - & - & - \\
\hline Ho & - & - & - & - & 0.86 & 0.91 & - & - & - \\
\hline Er & - & . & - & - & 2.25 & 2.36 & - & - & . \\
\hline $\mathrm{Yb}$ & - & 1.44 & - & 1.29 & 1.67 & 1.59 & - & - & - \\
\hline Lu & - & 0.20 & - & 0.18 & 0.26 & 0.24 & - & - & - \\
\hline \multicolumn{10}{|l|}{ Isotopic compositions } \\
\hline${ }^{87} \mathrm{Sr} / 86 \mathrm{Sr}$ (initial) & - & - & - & - & 0.70302 & - & - & - & - \\
\hline${ }^{143} \mathrm{Nd} / 144 \mathrm{Nd}$ (initial) & - & - & - & -( & 0.512782 & - & - & - & - \\
\hline $206 \mathrm{~Pb} / 204 \mathrm{~Pb}$ (measured) & - & - & - & - & 20.977 & - & - & - & - \\
\hline $207 \mathrm{~Pb} / 204 \mathrm{~Pb}$ (measured) & - & - & - & - & 15.718 & - & - & - & - \\
\hline${ }^{208} \mathrm{~Pb} / 204 \mathrm{~Pb}$ (measured) & - & - & - & - & 40.089 & - & - & - & - \\
\hline
\end{tabular}


Table 1 (continued).

\begin{tabular}{|c|c|c|c|c|c|c|c|c|c|c|c|}
\hline $800 \mathrm{~A}$ & $800 \mathrm{~A}$ & $800 \mathrm{~A}$ & $800 \mathrm{~A}$ & $800 \mathrm{~A}$ & $800 \mathrm{~A}$ & $800 \mathrm{~A}$ & $800 \mathrm{~A}$ & $800 \mathrm{~A}$ & $800 \mathrm{~A}$ & $800 \mathrm{~A}$ & $801 \mathrm{~B}$ \\
\hline 58R--2 & $58 \mathrm{R}-2$ & $58 \mathrm{R}-2$ & $58 \mathrm{R}-2$ & $58 \mathrm{R}-3$ & $59 \mathrm{R}-1$ & $60 \mathrm{R}-1$ & $60 \mathrm{R}-1$ & $60 \mathrm{R}-1$ & $61 \mathrm{R}-1$ & $61 R-1$ & $38 \mathrm{R}-1$ \\
\hline $67--73$ & $52--60$ & $106-110$ & $142--150$ & $35--41$ & 4--9 & $15--20$ & $44-46$ & 69--74 & $10--16$ & $19--24$ & $22--25$ \\
\hline 509.2 & 509.35 & 509.59 & 509.95 & 510.38 & 516.64 & 526.05 & 526.3 & 526.59 & 535.4 & 535.47 & 466.32 \\
\hline 3 & 3 & 3 & 3 & 3 & 3 & 3 & 3 & 3 & 3 & 3 & 3 \\
\hline 50.40 & 50.52 & 50.32 & 51.21 & 51.22 & 49.73 & 49.90 & 50.67 & 50.80 & 50.68 & 50.21 & $\overline{51.45}$ \\
\hline 2.13 & 2.23 & 2.03 & 2.04 & 2.14 & 2.17 & 2.12 & 2.14 & 2.09 & 2.12 & 2.29 & 2.67 \\
\hline 16.00 & 16.15 & 15.96 & 16.22 & 16.56 & 15.92 & 16.06 & 16.11 & 16.09 & 15.99 & 15.99 & 18.62 \\
\hline 4.89 & 4.71 & 6.47 & 4.87 & 4.14 & 4.50 & 4.33 & 5.27 & 5.66 & 4.89 & 4.99 & 5.97 \\
\hline 4.86 & 4.60 & 3.38 & 4.30 & 4.84 & 5.30 & 4.96 & 4.87 & 3.85 & 4.86 & 4.89 & 0.61 \\
\hline 0.15 & 0.18 & 0.16 & 0.19 & 0.21 & 0.16 & 0.14 & 0.16 & 0.14 & 0.12 & 0.12 & 0.04 \\
\hline 6.73 & 6.31 & 6.73 & 6.42 & 6.12 & 6.31 & 6.36 & 6.48 & 6.75 & 6.53 & 6.67 & 4.69 \\
\hline 4.89 & 4.74 & 3.92 & 4.75 & 5.20 & 5.44 & 5.94 & 5.00 & 4.64 & 5.26 & 5.05 & 2.00 \\
\hline 3.87 & 4.05 & 3.79 & 4.07 & 4.41 & 4.22 & 4.04 & 4.00 & 4.02 & 4.04 & 4.06 & 2.04 \\
\hline 2.77 & 2.76 & 2.93 & 2.92 & 2.24 & 1.94 & 2.29 & 2.50 & 2.49 & 2.29 & 2.33 & 5.80 \\
\hline 0.53 & 0.56 & 0.46 & 0.63 & 0.48 & 0.55 & 0.48 & 0.55 & 0.46 & 0.47 & 0.60 & 0.36 \\
\hline 2.77 & 2.93 & 3.54 & 2.62 & 2.15 & 3.65 & 3.17 & 2.51 & 3.23 & 2.59 & 3.04 & 5.67 \\
\hline 100.0 & 99.7 & 99.7 & 100.2 & 99.7 & 99.9 & 99.8 & 100.3 & 100.2 & 99.8 & 100.2 & 99.9 \\
\hline 0.38 & 0.34 & 0.18 & 0.16 & 0.12 & 0.43 & 0.95 & - & 0.16 & 0.29 & 0.20 & 0.71 \\
\hline 2.41 & 2.49 & 3.14 & 2.22 & 1.97 & 2.70 & 2.22 & - & 2.50 & 2.20 & 2.83 & 4.81 \\
\hline 359 & 402 & 421 & 413 & 316 & 391 & 308 & 380 & 419 & 331 & 371 & 457 \\
\hline - & - & - & - & - & - & - & - & - & - & - & - \\
\hline 120 & 82 & 81 & 93 & 326 & 38 & 114 & 107 & 64 & 333 & 48 & 194 \\
\hline 74 & 42 & 43 & 65 & 64 & 38 & 32 & 77 & 49 & 22 & 24 & 64 \\
\hline 24 & 22 & 24 & 23 & 24 & 21 & 22 & 23 & 24 & 21 & 21 & 18 \\
\hline 51 & 55 & 58 & 56 & 56 & 58 & 52 & 54 & 55 & 52 & 55 & 36 \\
\hline 44 & 39 & 43 & 43 & 42 & 39 & 43 & 44 & 44 & 43 & 39 & 67 \\
\hline 2 & 1 & 2 & 2 & 1 & 1 & 2 & 3 & 1 & 1 & 1 & 1 \\
\hline 25 & 23 & 27 & 29 & 28 & 21 & 25 & 27 & 24 & 25 & 23 & 82 \\
\hline 315 & 305 & 268 & 274 & 271 & 350 & 194 & 334 & 263 & 159 & 168 & 162 \\
\hline 548 & 544 & 473 & 526 & 597 & 574 & 585 & 559 & 518 & 572 & 542 & 102 \\
\hline 187 & 185 & 190 & 154 & 211 & 189 & 181 & 178 & 184 & 200 & 194 & 335 \\
\hline 25 & 25 & 24 & 25 & 25 & 25 & 24 & 27 & 24 & 25 & 26 & 20 \\
\hline 82 & 104 & 114 & 90 & 96 & 115 & 76 & 95 & 111 & 85 & 111 & 93 \\
\hline \multirow[t]{2}{*}{344} & 379 & 390 & 400 & 394 & 386 & 357 & 370 & 379 & 356 & 363 & 194 \\
\hline & WU & WU & & & & & WU & WU & & WU & WU \\
\hline- & 28 & 28 & - & - & - & - & 31 & 29 & - & 30 & 18 \\
\hline 0.10 & 0.06 & 0.1 & - & 0,1 & - & 0.11 & 0.09 & 0.07 & 0.1 & 0.11 & 1.33 \\
\hline 8.10 & 8.29 & 8.19 & - & 8.3 & - & 7.8 & 8.03 & 7.87 & 8.5 & 7.84 & 4.19 \\
\hline 19.10 & 18 & 17.1 & - & 19.3 & - & 17.4 & 19.4 & 17.4 & 20.6 & 5.6 & 28.20 \\
\hline 3.19 & 3.53 & 3.71 & - & 3.63 & - & 3.36 & 3.45 & 3.46 & 3.4 & 3.5 & 2.47 \\
\hline 4.10 & 4.06 & 4.22 & - & 4.6 & - & 4.4 & 3.99 & 3.98 & 4.4 & 4.06 & 2.00 \\
\hline 1.23 & 1.37 & 1.48 & - & 1.39 & - & 1.25 & 1.43 & 1.36 & 1.22 & 1.27 & 1.18 \\
\hline 43.60 & 41.4 & 39 & - & 45.99 & - & 43.9 & 39 & 36 & 42.37 & 40.4 & 20.30 \\
\hline 89.00 & 86.6 & 80.4 & - & 92.56 & - & 89.16 & 81.8 & 76.2 & 85.6 & 85.4 & 43.00 \\
\hline 10.06 & - & - & - & 10.53 & - & 9.95 & - & - & 9.78 & - & . \\
\hline 40.34 & 38 & 37 & - & 41.51 & - & 39.71 & 40 & 36 & 38.99 & 41 & 21.00 \\
\hline 7.80 & 8.34 & 7.58 & - & 7.85 & - & 7.52 & 7.96 & 7.51 & 7.42 & 8.36 & 4.17 \\
\hline 2.58 & 2.64 & 2.38 & - & 2.57 & - & 2.5 & 2.53 & 2.43 & 2.51 & 2.6 & 1.32 \\
\hline 6.87 & - & - & - & 6.85 & - & 6.57 & - & - & 6.69 & . & . \\
\hline - & 0.95 & 0.88 & - & - & - & - & 0.95 & 0.88 & - & 0.96 & 0.61 \\
\hline 5.27 & - & - & - & 5.37 & - & 5.22 & - & - & 5.26 & - & . \\
\hline 0.86 & - & - & - & 0.88 & - & 0.85 & . & . & 0.86 & . & . \\
\hline 2.25 & - & - & - & 2.36 & - & 2.29 & - & - & 2.31 & - & - \\
\hline 1.55 & 1.5 & 1.48 & - & 1.67 & - & 1.63 & 1.52 & 1.42 & 1.63 & 1.57 & 1.69 \\
\hline 0.24 & 0.2 & 0.2 & - & 0.26 & - & 0.26 & 0.22 & 0.2 & 0.26 & 0.22 & 0.24 \\
\hline - & 0.70320 & - & - & - & - & - & . & 0.70292 & - & 0.70300 & . \\
\hline - & 0.512781 & - & - & - & - & - & - & 0.512784 & - & 0.512806 & - \\
\hline - & 20.988 & - & - & - & - & - & - & 20.525 & - & 21.451 & . \\
\hline - & 15.722 & - & - & - & - & - & - & 15.700 & - & 15.767 & . \\
\hline - & 40.258 & - & - & - & - & - & - & 40.015 & - & 40.682 & . \\
\hline
\end{tabular}


Table 1 (continued).

\begin{tabular}{|c|c|c|c|c|c|c|c|c|c|c|c|}
\hline $801 \mathrm{~B}$ & $801 \mathrm{~B}$ & $801 B$ & $801 \mathrm{~B}$ & $801 \mathrm{~B}$ & 801B & $801 B$ & $801 \mathrm{~B}$ & $801 \mathrm{~B}$ & $801 \mathrm{~B}$ & $801 \mathrm{~B}$ & $801 \mathrm{~B}$ \\
\hline $40 \mathrm{R}-1$ & $40 \mathrm{R}-1$ & $40 \mathrm{R}-1$ & $41 \mathrm{R}-1$ & $41 \mathrm{R}-1$ & $41 R-1$ & $41 \mathrm{R}-1$ & $41 R-2$ & $42 \mathrm{R}-1$ & $42 \mathrm{R}-1$ & $42 R-2$ & $42 R-2$ \\
\hline $15--18$ & $29--33$ & $85--91$ & $26--31$ & $88--94$ & $130--136$ & $20--25$ & $103-109$ & $24--30$ & $87--91$ & $0--7$ & $129--134$ \\
\hline 477.05 & 477.19 & 477.75 & 483.26 & 483.88 & 484.3 & 484.7 & 485.53 & 487.84 & 488.47 & 489.05 & 490.34 \\
\hline 4 & 4 & 4 & 5 & 5 & 5 & 5 & 5 & 6 & 6 & 6 & 6 \\
\hline 51.58 & 53.20 & 39.67 & 45.77 & 46.36 & 46.08 & 46.33 & 46.36 & 47.08 & 46.50 & 46.91 & 46.33 \\
\hline 3.30 & 3.58 & 2.70 & 3.31 & 3.27 & 3.24 & 3.56 & 3.07 & 3.03 & 3.01 & 2.89 & 2.92 \\
\hline 17.42 & 18.96 & 14.02 & 16.03 & 15.89 & 15.80 & 15.14 & 16.61 & 17.32 & 17.23 & 17.42 & 16.96 \\
\hline 9.02 & 5.54 & 3.57 & 4.76 & 3.80 & 4.28 & 4.27 & 4.32 & 4.91 & 5.04 & 4.63 & 4.04 \\
\hline 0.75 & 0.77 & 3.92 & 4.72 & 5.37 & 5.79 & 5.57 & 5.40 & 4.39 & 4.31 & 4.68 & 5.70 \\
\hline 0.12 & 0.08 & 0.23 & 0.14 & 0.13 & 0.15 & 0.14 & 0.14 & 0.14 & 0.14 & 0.14 & 0.14 \\
\hline 2.75 & 2.87 & 4.11 & 5.20 & 5.07 & 4.86 & 4.52 & 4.79 & 5.68 & 6.26 & 6.50 & 7.18 \\
\hline 2.63 & 1.88 & 14.27 & 8.25 & 7.82 & 7.64 & 7.71 & 7.76 & 8.29 & 8.26 & 8.36 & 8.06 \\
\hline 2.91 & 2.76 & 3.83 & 3.81 & 3.64 & 3.71 & 3.88 & 3.60 & 3.64 & 3.49 & 3.43 & 3.26 \\
\hline 5.88 & 5.84 & 1.74 & 2.14 & 2.45 & 2.61 & 3.01 & 2.54 & 2.02 & 1.97 & 1.96 & 1.82 \\
\hline 0.63 & 0.67 & 0.56 & 0.62 & 0.69 & 0.61 & 0.69 & 0.59 & 0.59 & 0.56 & 0.54 & 0.56 \\
\hline 2.68 & 4.12 & 10.92 & 5.09 & 4.72 & 4.71 & 4.67 & 4.95 & 3.14 & 3.34 & 2.93 & 2.85 \\
\hline 99.7 & 100.3 & 99.5 & 99.8 & 99.2 & 99.5 & 99.5 & 100.1 & 100.2 & 100.1 & 100.4 & 99.8 \\
\hline 0.11 & 0.24 & 9.45 & 2.83 & 2.76 & 3.00 & 2.90 & 2.96 & 0.97 & 0.97 & 1.07 & 0.85 \\
\hline 2.72 & 3.77 & 1.54 & 2.00 & 1.60 & 1.55 & 1.54 & 1.86 & 2.04 & 2.32 & 1.97 & 2.09 \\
\hline 439 & 406 & 318 & 414 & 452 & 422 & 448 & 395 & 386 & 365 & 377 & 354 \\
\hline - & - & - & - & - & - & - & - & - & - & - & - \\
\hline 123 & 89 & 93 & 73 & 48 & 61 & 42 & 113 & 137 & 134 & 154 & 180 \\
\hline 37 & 20 & 24 & 44 & 47 & 44 & 44 & 40 & 40 & 42 & 37 & 39 \\
\hline 22 & 26 & 23 & 23 & 21 & 24 & 23 & 23 & 23 & 23 & 22 & 20 \\
\hline 49 & 51 & 40 & 48 & 48 & 49 & 53 & 44 & 44 & 44 & 42 & 41 \\
\hline 82 & 66 & 43 & 46 & 47 & 45 & 28 & 50 & 71 & 72 & 69 & 92 \\
\hline 6 & 1 & 9 & 3 & 2 & 10 & 9 & 8 & 3 & 2 & 8 & 7 \\
\hline 110 & 88 & 33 & 39 & 48 & 52 & 62 & 52 & 39 & 40 & 42 & 38 \\
\hline 105 & 146 & 224 & 358 & 293 & 250 & 251 & 239 & 368 & 370 & 276 & 298 \\
\hline 317 & 134 & 654 & 518 & 503 & 512 & 486 & 526 & 571 & 548 & 572 & 559 \\
\hline 241 & 198 & 235 & 267 & 261 & 275 & 301 & 252 & 236 & 230 & 234 & 218 \\
\hline 33 & 27 & 30 & 32 & 32 & 32 & 36 & 30 & 29 & 29 & 29 & 28 \\
\hline 111 & 74 & 64 & 90 & 89 & 90 & 101 & 85 & 89 & 79 & 72 & 71 \\
\hline 153 & 208 & 229 & 272 & 280 & 285 & 308 & 256 & 247 & 246 & 243 & 233 \\
\hline WU & & WU & WU & & & & & & & & WU \\
\hline 36 & - & 25 & 28 & - & - & - & - & - & - & - & 34 \\
\hline 3.31 & - & 0.31 & 0.29 & - & 0.39 & 0.47 & - & - & - & 0.29 & 0.24 \\
\hline 3.64 & - & 5.34 & 5.92 & - & 6.40 & 7.50 & - & - & - & 5.80 & 5.30 \\
\hline 11.69 & - & 18.60 & 22.50 & - & 25.20 & 28.60 & - & - & . & 22.60 & 20.10 \\
\hline 3.17 & - & 2.69 & 3.17 & - & 3.23 & 3.49 & - & - & - & 2.76 & 2.80 \\
\hline 3.16 & - & 2.58 & 3.02 & - & 3.50 & 3.60 & - & - & - & 3.00 & 2.57 \\
\hline 0.78 & - & 1.39 & 1.40 & - & 1.01 & 1.04 & - & - & - & 1.00 & 0.89 \\
\hline 30.10 & - & 29.70 & 30.50 & - & 36.30 & 39.61 & - & - & - & 32.44 & 27.60 \\
\hline 59.10 & - & 61.30 & 63.80 & - & 74.16 & 81.76 & - & - & - & 64.95 & 58.40 \\
\hline - & - & - & - & - & 8.65 & 9.36 & - & - & - & 7.48 & 20.00 \\
\hline 28.00 & - & 29.00 & 31.00 & - & 35.47 & 39.08 & - & - & - & 31.41 & 25.00 \\
\hline 6.88 & - & 6.80 & 7.28 & - & 7.41 & 8.22 & - & - & - & 6.62 & 6.62 \\
\hline 2.17 & - & 2.15 & 2.32 & - & 2.47 & 2.69 & - & - & - & 2.22 & 2.09 \\
\hline - & - & - & - & - & 7.23 & 8.05 & - & - & . & 6.58 & - \\
\hline 0.93 & - & 0.97 & 1.03 & - & - & - & - & - & - & - & 0.89 \\
\hline- & - & - & - & - & 6.40 & 7.00 & - & - & . & 5.68 & - \\
\hline- & - & - & - & - & 1.14 & 1.22 & - & - & - & 1.02 & - \\
\hline- & - & - & - & - & 3.11 & 3.36 & - & - & - & 2.71 & - \\
\hline 1.84 & - & 2.20 & 2.31 & - & 2.60 & 2.82 & - & - & - & 2.26 & 2.18 \\
\hline 0.25 & - & 0.31 & 0.32 & - & 0.44 & 0.44 & $\cdot$ & - & - & 0.38 & 0.30 \\
\hline- & - & - & 0.70276 & - & - & - & - & - & - & - & - \\
\hline - & - & - & 0.512848 & - & - & - & - & - & - & - & - \\
\hline - & - & - & 19.300 & - & - & - & - & - & - & - & - \\
\hline - & - & - & 15.589 & $\cdot$ & - & - & - & - & - & - & - \\
\hline- & - & - & 38.569 & - & - & - & - & - & - & - & - \\
\hline
\end{tabular}


Table 1 (continued).

\begin{tabular}{|c|c|c|c|c|c|c|c|c|c|c|c|}
\hline $801 \mathrm{~B}$ & $801 \mathrm{~B}$ & $801 B$ & $801 \mathrm{~B}$ & $801 \mathrm{~B}$ & $801 \mathrm{~B}$ & $801 B$ & $801 B$ & $801 \mathrm{~B}$ & $801 B$ & $801 \mathrm{~B}$ & $801 B$ \\
\hline 43R-1 & $43 R-1$ & $43 \mathrm{R}-1$ & 43R-1 & $43 R-2$ & $43 R-2$ & $43 R-3$ & $43 R-3$ & $43 R-4$ & $43 R-4$ & $44 \mathrm{R}-1$ & $44 \mathrm{R}-1$ \\
\hline 4--9 & $16--20$ & $22--27$ & $128--134$ & $10-15$ & $21--27$ & $23--27$ & $126-129$ & $5-10$ & $32-40$ & $18--24$ & $107-113$ \\
\hline 492.24 & 492.36 & 492.42 & 493.48 & 493.67 & 493.78 & 495.21 & 496.24 & 496.53 & 496.8 & 501.88 & 502.77 \\
\hline 7 & 7 & 7 & 7 & 7 & 7 & 7 & 9 & 10 & 11 & 12 & 12 \\
\hline 46.44 & 44.86 & 45.88 & 44.43 & 44.88 & 44.38 & 47.46 & 42.82 & 47.20 & 45.22 & 44.87 & 48.13 \\
\hline 2.97 & 2.76 & 2.94 & 2.48 & 2.68 & 2.50 & 3.32 & 2.85 & 3.08 & 3.38 & 3.26 & 3.26 \\
\hline 17.04 & 14.82 & 15.23 & 13.11 & 14.46 & 13.67 & 17.48 & 15.40 & 16.83 & 17.32 & 16.80 & 17.72 \\
\hline 4.25 & 4.52 & 4.70 & 5.73 & 4.86 & 4.95 & 3.67 & 3.20 & 4.11 & 5.20 & 3.76 & 4.32 \\
\hline 5.43 & 5.78 & 5.70 & 5.72 & 6.20 & 5.16 & 5.53 & 3.84 & 4.59 & 5.20 & 4.16 & 3.22 \\
\hline 0.13 & 0.14 & 0.13 & 0.15 & 0.16 & 0.16 & 0.20 & 0.49 & 0.20 & 0.20 & 0.31 & 0.18 \\
\hline 6.98 & 8.49 & 8.28 & 10.58 & 9.01 & 8.93 & 3.98 & 3.31 & 3.58 & 4.09 & 2.65 & 3.05 \\
\hline 7.83 & 7.01 & 6.21 & 6.59 & 6.74 & 7.38 & 3.38 & 9.01 & 6.15 & 6.24 & 9.37 & 4.35 \\
\hline 3.42 & 3.82 & 4.02 & 2.76 & 3.35 & 3.12 & 2.78 & 2.89 & 3.84 & 4.61 & 4.01 & 3.12 \\
\hline 1.88 & 1.79 & 1.90 & 1.46 & 1.79 & 1.63 & 4.95 & 4.55 & 3.27 & 2.34 & 2.94 & 4.57 \\
\hline 0.55 & 0.57 & 0.55 & 0.46 & 0.50 & 0.51 & 0.64 & 0.52 & 0.59 & 0.68 & 0.57 & 0.57 \\
\hline 2.70 & 5.14 & 4.58 & 6.16 & 5.21 & 7.22 & 6.22 & 10.63 & 6.82 & 5.85 & 7.69 & 6.96 \\
\hline 99.6 & 99.7 & 100.1 & 99.6 & 99.8 & 99.6 & 99.6 & 99.5 & 100.3 & 100.3 & 100.4 & 99.5 \\
\hline 0.76 & 0.86 & 0.35 & 2.46 & 2.02 & 3.94 & 3.05 & 8.69 & 4.20 & 2.36 & 5.50 & 3.24 \\
\hline 2.06 & 3.78 & 4.17 & 3.27 & 2.91 & 2.42 & 2,91 & 2.30 & 2.14 & 3.26 & 1.57 & 3.34 \\
\hline 375 & 517 & 495 & 371 & 483 & 460 & 481 & 413 & 452 & 547 & 376 & 437 \\
\hline - & - & - & - & - & - & - & - & - & - & - & \\
\hline 141 & 189 & 199 & 323 & 244 & 269 & 139 & 78 & 110 & 102 & 90 & 123 \\
\hline 39 & 41 & 39 & 35 & 33 & 35 & 37 & 36 & 33 & 40 & 35 & 35 \\
\hline 22 & 17 & 18 & 16 & 21 & 16 & 23 & 20 & 21 & 21 & 22 & 24 \\
\hline 43 & 39 & 42 & 37 & 40 & 35 & 49 & 40 & 43 & 40 & 43 & 42 \\
\hline 81 & 190 & 163 & 375 & 241 & 336 & 112 & 64 & 41 & 67 & 60 & 107 \\
\hline 8 & 2 & 4 & 2 & 8 & 2 & 11 & 9 & 6 & 5 & 9 & 1 \\
\hline 41 & 37 & 40 & 32 & 39 & 33 & 67 & 58 & 59 & 44 & 51 & 63 \\
\hline 283 & 333 & 379 & 1388 & 362 & 403 & 288 & 266 & 234 & 438 & 214 & 347 \\
\hline 564 & 509 & 384 & 372 & 413 & 399 & 266 & 243 & 411 & 404 & 490 & 250 \\
\hline 230 & 214 & 237 & 204 & 202 & 206 & 277 & 212 & 270 & 258 & 254 & 284 \\
\hline 29 & 27 & 28 & 25 & 27 & 25 & 28 & 31 & 29 & 32 & 32 & 27 \\
\hline 76 & 86 & 86 & 89 & 93 & 80 & 104 & 72 & 81 & 146 & 79 & 87 \\
\hline \multirow[t]{2}{*}{245} & 230 & 244 & 208 & 227 & 209 & 277 & 218 & 259 & 246 & 264 & 214 \\
\hline & & WU & & & & & & WU & & & WU \\
\hline - & - & 46 & - & - & - & - & - & 23 & - & - & 43 \\
\hline - & - & 0.35 & 0.28 & - & - & - & - & 0.32 & - & 0.50 & 0.52 \\
\hline - & - & 5.48 & 4.80 & - & - & - & - & 5.75 & - & 6.20 & 5.27 \\
\hline - & - & 21.10 & 20.70 & - & . & . & - & 20.00 & - & 23.80 & 181.00 \\
\hline - & - & 2.73 & 2.37 & - & - & - & - & 2.79 & . & 2.81 & 2.80 \\
\hline - & - & 2.56 & 2.54 & - & - & - & - & 2.65 & - & 2.80 & 2.62 \\
\hline - & - & 0.95 & 0.77 & - & - & . & - & 0.80 & - & 0.95 & 0.55 \\
\hline - & - & 27.10 & 27.56 & - & - & - & - & 27.90 & . & 32.84 & 27.00 \\
\hline- & - & 57.80 & 55.87 & - & - & - & - & 59.40 & - & 67.53 & 56.60 \\
\hline - & - & - & 63.10 & - & - & - & - & - & - & 7.88 & \\
\hline- & - & 28.00 & 27.17 & - & - & - & - & 24.00 & - & 32.94 & 25.00 \\
\hline - & - & 6.65 & 5.68 & - & - & - & - & 6.72 & - & 7.02 & 6.18 \\
\hline - & - & 2.11 & 1.89 & - & - & - & - & 2.16 & - & 2.37 & 2.06 \\
\hline - & - & - & 5.57 & - & - & - & - & - & - & 7.15 & \\
\hline - & - & 0.90 & - & - & - & - & - & 0.95 & - & - & 0.88 \\
\hline - & - & - & 4.91 & - & - & - & - & - & - & 6.25 & \\
\hline - & - & - & 0.85 & - & - & - & - & . & - & 1.12 & \\
\hline- & - & - & 2.36 & - & - & - & - & - & - & 3.03 & \\
\hline - & - & 2.12 & 1.97 & - & - & - & - & 2.33 & . & 2.56 & 2.02 \\
\hline - & - & 0.30 & 0.32 & - & - & - & - & 0.34 & - & 0.40 & 0.27 \\
\hline - & - & 0.70282 & - & - & - & - & - & - & - & - & \\
\hline- & - & 0.512814 & - & - & - & - & - & - & - & - & \\
\hline - & - & 19.223 & - & - & - & - & - & - & - & - & \\
\hline - & - & 15.556 & - & - & - & - & - & - & - & - & \\
\hline - & - & 38.413 & - & - & - & - & - & - & - & - & \\
\hline
\end{tabular}


Table 1 (continued).

\begin{tabular}{|c|c|c|c|c|c|c|c|c|c|c|c|}
\hline $801 \mathrm{~B}$ & $801 \mathrm{~B}$ & $801 B$ & 801B & 801B & $801 C$ & $801 C$ & $801 \mathrm{C}$ & $801 C$ & $801 C$ & $801 C$ & $801 \mathrm{C}$ \\
\hline $44 \mathrm{R}-2$ & $44 R-2$ & $44 \mathrm{R}-2$ & $44 \mathrm{R}-3$ & $44 \mathrm{R}-3$ & $1 \mathrm{R}-1$ & $1 \mathrm{R}-1$ & 1R-1 & $1 R-2$ & $1 \mathrm{R}-3$ & IR-3 & $1 R-4$ \\
\hline $27--32$ & $67--72$ & $140--145$ & $13--22$ & $28--33$ & $14--20$ & $109--114$ & $120-125$ & $11--17$ & $72--76$ & $103--106$ & $0--5$ \\
\hline 503.42 & 503.82 & 504.55 & 504.76 & 504.91 & 493.84 & 494.79 & 494.9 & 495.06 & 497.03 & 497.34 & 497.4 \\
\hline \multicolumn{12}{|c|}{$\mathrm{UM}$} \\
\hline 51.42 & 45.67 & 48.57 & 49.06 & 47.65 & 44.06 & 44.92 & 44.60 & 45.17 & 43.37 & 47.70 & 47.20 \\
\hline 3.27 & 3.12 & 3.31 & 3.25 & 3.16 & 2.69 & 2.80 & 2.66 & 2.81 & 3.00 & 3.24 & 3.19 \\
\hline 17.70 & 17.02 & 17.46 & 17.87 & 17.22 & 14.18 & 14.72 & 14.60 & 14.87 & 15.99 & 17.11 & 16.95 \\
\hline 3.75 & 4.28 & 4.48 & 4.19 & 4.18 & 4.49 & 3.62 & 3.86 & 4.10 & 4.50 & 3.76 & 3.57 \\
\hline 3.27 & 4.38 & 4.46 & 4.99 & 5.04 & 6.34 & 6.40 & 5.50 & 6.30 & 6.60 & 4.48 & 4.52 \\
\hline 0.13 & 0.20 & 0.13 & 0.11 & 0.14 & 0.16 & 0.15 & 0.14 & 0.15 & 0.20 & 0.19 & 0.28 \\
\hline 3.03 & 3.99 & 4.08 & 3.69 & 4.21 & 10.14 & 9.58 & 8.34 & 9.34 & 6.54 & 4.22 & 2.77 \\
\hline 3.56 & 7.95 & 7.02 & 6.47 & 7.24 & 7.44 & 6.46 & 6.48 & 6.34 & 6.53 & 5.00 & 5.73 \\
\hline 3.20 & 4.21 & 4.23 & 4.47 & 4.08 & 2.58 & 3.45 & 3.42 & 3.63 & 3.14 & 3.56 & 3.41 \\
\hline 5.67 & 1.88 & 1.88 & 2.15 & 2.31 & 1.57 & 1.75 & 1.83 & 1.82 & 2.36 & 4.22 & 4.47 \\
\hline 0.55 & 0.52 & 0.57 & 0.74 & 0.57 & 0.51 & 0.60 & 0.52 & 0.53 & 0.57 & 0.58 & 0.59 \\
\hline 4.38 & 6.79 & 4.35 & 3.46 & 3.65 & 5.31 & 5.30 & 6.23 & 5.01 & 6.89 & 5.45 & 6.94 \\
\hline 99.9 & 100.0 & 100.5 & 100.5 & 99.5 & 99.5 & 99.8 & 98.8 & 100.1 & 99.7 & 99.5 & 99.6 \\
\hline 2.20 & 4.36 & 2.69 & - & 2.05 & 2.64 & 1.15 & 0.83 & 1.37 & 3.12 & - & 4.42 \\
\hline \multirow[t]{2}{*}{2.06} & 1.95 & 1.40 & - & 1.40 & 2.42 & 3.77 & 2.70 & 3.44 & 3.44 & - & 2.10 \\
\hline & & & \multicolumn{9}{|c|}{ UM } \\
\hline \multirow[t]{2}{*}{531} & 353 & 375 & 434 & 396 & 315 & 427 & 410 & 493 & 385 & 689 & 489 \\
\hline & - & - & - & - & - & - & 47 & - & - & - & - \\
\hline 130 & 101 & 170 & 105 & 92 & 227 & 154 & 164 & 160 & 173 & 99 & 92 \\
\hline 42 & 42 & 41 & 48 & 36 & 39 & 40 & 32 & 40 & 42 & 34 & 49 \\
\hline 20 & 22 & 22 & 23 & 22 & 20 & 19 & - & 19 & 21 & 24 & 25 \\
\hline 44 & 40 & 41 & 44 & 44 & 38 & 41 & 52 & 42 & 45 & 45 & 45 \\
\hline 93 & 65 & 41 & 58 & 59 & 247 & 197 & 204 & 212 & 133 & 59 & 74 \\
\hline 8 & 7 & 6 & 3 & 10 & 1 & 1 & - & 3 & 5 & 2 & 3 \\
\hline 67 & 38 & 36 & 42 & 46 & 35 & 40 & 66 & 41 & 46 & 61 & 62 \\
\hline 264 & 257 & 229 & 265 & 285 & 381 & 263 & - & 261 & 386 & 170 & 222 \\
\hline 311 & 526 & 568 & 567 & 528 & 465 & 363 & 371 & 360 & 368 & 378 & 349 \\
\hline 253 & 239 & 293 & 276 & 243 & 224 & 241 & - & 205 & 290 & 249 & 264 \\
\hline 31 & 31 & 29 & 37 & 33 & 27 & 27 & 29 & 28 & 31 & 32 & 32 \\
\hline 69 & 85 & 77 & 64 & 83 & 84 & 86 & 86 & 85 & 119 & 68 & 88 \\
\hline \multirow[t]{2}{*}{254} & 251 & 261 & 278 & 268 & 221 & 242 & 235 & 239 & 262 & 244 & 279 \\
\hline & & & & WU & & WU & & & & & \\
\hline - & - & - & - & 35 & - & 46 & - & - & - & - & - \\
\hline 0.74 & - & - & - & 0.33 & 0.31 & 0.31 & - & - & - & - & - \\
\hline 5.80 & - & - & - & 5.88 & 4.90 & 5.40 & - & - & - & - & - \\
\hline 23.70 & - & - & - & 20.90 & 20.60 & 20.10 & - & - & - & - & - \\
\hline 2.80 & - & - & - & 2.79 & 2.31 & 2.62 & - & - & - & - & - \\
\hline 2.80 & - & - & - & 2.76 & 2.50 & 2.61 & - & $\cdot$ & - & - & - \\
\hline 2.30 & - & - & - & 0.97 & 0.83 & 0.97 & - & . & - & - & - \\
\hline 31.84 & - & - & - & 28.90 & 28.97 & 27.40 & - & - & - & - & - \\
\hline 66.47 & - & - & - & 60.60 & 59.22 & 57.50 & - & - & - & - & - \\
\hline 7.68 & - & - & - & - & 6.69 & - & - & - & - & - & - \\
\hline 32.40 & - & - & - & 33.00 & 28.43 & 30.00 & - & - & - & - & - \\
\hline 6.97 & - & - & - & 7.09 & 6.06 & 6.37 & - & - & - & - & - \\
\hline 2.31 & - & - & - & 2.33 & 1.99 & 2.05 & - & - & - & - & - \\
\hline 6.96 & - & - & - & - & 5.95 & - & - & - & - & - & - \\
\hline - & - & - & - & 1.09 & - & 0.90 & - & - & - & - & - \\
\hline 6.14 & - & - & - & - & 5.18 & - & - & - & - & - & - \\
\hline 1.08 & - & - & - & - & 0.90 & - & - & . & - & - & - \\
\hline 2.99 & - & - & - & - & 2.50 & - & - & - & - & - & - \\
\hline 2.52 & - & . & - & 2.60 & 2.08 & 2.05 & - & - & - & - & - \\
\hline 0.40 & - & - & - & 0.38 & 0.34 & 0.30 & - & $\cdot$ & - & - & - \\
\hline - & - & - & - & 0.70287 & - & 0.70283 & - & - & - & - & - \\
\hline - & - & - & - & 0.512905 & - & 0.512829 & - & - & - & - & - \\
\hline - & - & - & - & 18.951 & - & 19.215 & - & - & - & - & - \\
\hline - & - & - & - & 15.545 & - & 15.563 & - & - & - & - & - \\
\hline - & - & - & - & 38.167 & - & 38.417 & - & - & - & - & - \\
\hline
\end{tabular}


Table 1 (continued).

\begin{tabular}{|c|c|c|c|c|c|c|c|c|c|c|c|}
\hline $801 C$ & $801 C$ & $801 \mathrm{C}$ & $801 C$ & $801 C$ & $801 \mathrm{C}$ & $801 C$ & $801 C$ & $801 C$ & $801 C$ & $801 C$ & $801 C$ \\
\hline $1 \mathrm{R}-5$ & $1 \mathrm{R}-5$ & 1R-6 & 2R-1 & $2 R-1$ & $2 R-2$ & $2 R-3$ & $2 R-3$ & $2 R-4$ & $2 R-5$ & $3 R-1$ & $3 R-1$ \\
\hline $33--38$ & $113--118$ & $65--70$ & $1--6$ & $112-118$ & $131--137$ & $105--110$ & $114-119$ & $71--76$ & $101-106$ & $14-20$ & $42--48$ \\
\hline 498.88 & 499.68 & 500.62 & 503 & 504.12 & 505.69 & 506.89 & 506.98 & 507.81 & 509.5 & 512.34 & 512.63 \\
\hline \multirow[t]{2}{*}{2} & 2 & 2 & 3 & 3 & 3 & 4 & 6 & 6 & 6 & 7 & 7 \\
\hline & & UM & & & & & & & \multicolumn{3}{|c|}{ UM } \\
\hline 46.13 & 46.20 & 41.20 & 51.99 & 45.53 & 46.22 & 47.61 & 45.25 & 45.95 & 46.00 & 46.40 & 52.83 \\
\hline 3.14 & 2.93 & 2.68 & 3.34 & 3.07 & 2.96 & 3.26 & 3.17 & 3.15 & 3.13 & 2.89 & 3.53 \\
\hline 16.57 & 16.72 & 15.10 & 17.94 & 16.31 & 16.42 & 17.49 & 16.77 & 16.40 & 16.81 & 16.50 & 18.77 \\
\hline 3.96 & 3.38 & 4.09 & 3.63 & 4.25 & 4.14 & 3.62 & 3.73 & 3.66 & 3.99 & 7.45 & 5.09 \\
\hline 5.79 & 5.71 & 5.50 & 3.01 & 5.32 & 5.74 & 4.65 & 5.27 & 6.19 & 6.08 & 0.90 & 1.00 \\
\hline 0.16 & 0.16 & 0.29 & 0.13 & 0.16 & 0.17 & 0.18 & 0.19 & 0.15 & 0.16 & 0.06 & 0.05 \\
\hline 5.08 & 5.15 & 5.83 & 2.62 & 4.68 & 6.23 & 3.86 & 4.90 & 5.28 & 6.16 & 3.12 & 3.32 \\
\hline 6.59 & 6.15 & 7.28 & 2.35 & 7.14 & 7.06 & 6.01 & 6.34 & 7.13 & 6.78 & 5.09 & 1.94 \\
\hline 4.63 & 4.74 & 2.81 & 2.53 & 3.94 & 3.53 & 4.01 & 3.98 & 3.86 & 3.67 & 2.52 & 2.28 \\
\hline 2.43 & 2.25 & 1.95 & 6.40 & 2.14 & 2.05 & 2.56 & 2.45 & 2.33 & 2.20 & 4.30 & 5.68 \\
\hline 0.57 & 0.61 & 0.50 & 0.60 & 0.61 & 0.52 & 0.58 & 0.60 & 0.54 & 0.53 & 0.52 & 0.62 \\
\hline 4.36 & 5.75 & 11.30 & 4.76 & 6.92 & 5.16 & 6.04 & 7.39 & 5.82 & 4.62 & 9.47 & 4.58 \\
\hline 99.4 & 99.8 & 99.1 & 99.3 & 100.1 & 100.2 & 99.9 & 100.0 & 100.5 & 100.1 & 99.3 & 99.7 \\
\hline 1.48 & 2.34 & 5.88 & 1.72 & 4.53 & 3.04 & 3.71 & 4.68 & 4.23 & 2.70 & 2.45 & 0.41 \\
\hline \multirow[t]{2}{*}{2.90} & 2.78 & 2.80 & 2.92 & 1.86 & 1.79 & 1.80 & 2.13 & 1.36 & 1.77 & 3.70 & 4.00 \\
\hline & & UM & & & & & & & \multicolumn{3}{|c|}{ UM } \\
\hline 948 & 633 & 331 & 500 & 396 & 355 & 398 & 408 & 387 & 412 & 432 & 424 \\
\hline - & - & 52 & - & - & - & - & - & - & - & 34 & - \\
\hline 84 & 78 & 194 & 131 & 87 & 170 & 226 & 131 & 76 & 84 & 125 & 155 \\
\hline 36 & 36 & 31 & 37 & 40 & 36 & 42 & 42 & 40 & 44 & 43 & 55 \\
\hline 20 & 24 & - & 20 & 24 & 21 & 24 & 24 & 23 & 22 & - & 24 \\
\hline 44 & 41 & 47 & 45 & 42 & 39 & 43 & 41 & 41 & 40 & 55 & 46 \\
\hline 57 & 57 & 203 & 119 & 42 & 109 & 116 & 114 & 46 & 56 & 115 & $99^{\circ}$ \\
\hline 5 & 1 & - & 2 & 3 & 2 & 1 & 1 & 2 & 1 & - & 1 \\
\hline 47 & 47 & 49 & 66 & 44 & 41 & 50 & 48 & 46 & 42 & 70 & 71 \\
\hline 312 & 351 & - & 231 & 307 & 262 & 341 & 279 & 246 & 247 & - & 120 \\
\hline 470 & 358 & 436 & 160 & 477 & 499 & 507 & 498 & 495 & 499 & 255 & 112 \\
\hline 248 & 249 & - & 279 & 254 & 256 & 267 & 265 & 262 & 241 & - & 284 \\
\hline 32 & 29 & 16 & 35 & 31 & 30 & 32 & 30 & 32 & 31 & 31 & 32 \\
\hline 76 & 86 & 73 & 54 & 83 & 87 & 74 & 78 & 87 & 78 & 90 & 85 \\
\hline 267 & 261 & 222 & 258 & 259 & 250 & 270 & 255 & 257 & 255 & 249 & 248 \\
\hline WU & & & & WU & & & WU & & & & \\
\hline 30 & - & - & - & 28 & - & - & 41 & - & - & - & - \\
\hline 0.30 & - & - & - & 0.44 & - & - & 0.42 & 0.4 & - & - & - \\
\hline 5.91 & - & - & - & 5.7 & - & - & 5.74 & 5.7 & - & - & - \\
\hline 20.60 & - & - & - & 21.5 & - & - & 19.7 & 20.9 & - & - & - \\
\hline 2.86 & - & - & - & 2.46 & - & - & 2.64 & 2.45 & - & - & - \\
\hline 2.69 & - & - & - & 2.43 & - & - & 2.47 & 2.46 & - & - & - \\
\hline 1.06 & - & - & - & 0.81 & - & - & 1.14 & 0.85 & - & - & - \\
\hline 28.90 & - & - & - & 31.16 & - & - & 27.7 & 31.14 & - & - & - \\
\hline 60.30 & - & - & - & 64.62 & - & - & 58 & 64.75 & - & - & - \\
\hline - & - & - & - & 7.49 & - & - & - & 7.45 & - & . & . \\
\hline 31.00 & - & - & - & 31.68 & - & - & 25 & 31.41 & - & - & - \\
\hline 7.02 & - & - & $:$ & 6.93 & - & - & 6.73 & 6.85 & - & - & - \\
\hline 2.30 & - & - & - & 2.32 & - & - & 2.21 & 2.29 & - & - & - \\
\hline - & - & - & - & 6.9 & - & - & - & 6.88 & - & - & - \\
\hline 1.03 & - & - & - & - & - & - & 0.93 & - & - & - & - \\
\hline - & $\cdot$ & - & - & 6.21 & - & - & - & 6.16 & - & - & - \\
\hline - & - & - & - & 1.09 & - & - & - & 1.09 & - & - & - \\
\hline$\therefore$ & - & - & - & 2.99 & - & - & - & 3 & - & - & - \\
\hline 2.51 & - & - & - & 2.58 & - & - & 2.25 & 2.56 & - & - & - \\
\hline 0.36 & $\cdot$ & - & - & 0.42 & - & - & 0.32 & 0.41 & - & - & $\therefore$ \\
\hline - & - & - & - & - & - & - & - & - & - & - & - \\
\hline- & $\cdot$ & - & - & - & - & - & - & - & - & - & - \\
\hline - & - & - & - & - & - & - & - & - & - & - & - \\
\hline- & - & - & - & - & - & - & - & - & - & - & - \\
\hline - & - & - & - & - & - & - & - & - & - & - & - \\
\hline
\end{tabular}


Table 1 (continued).

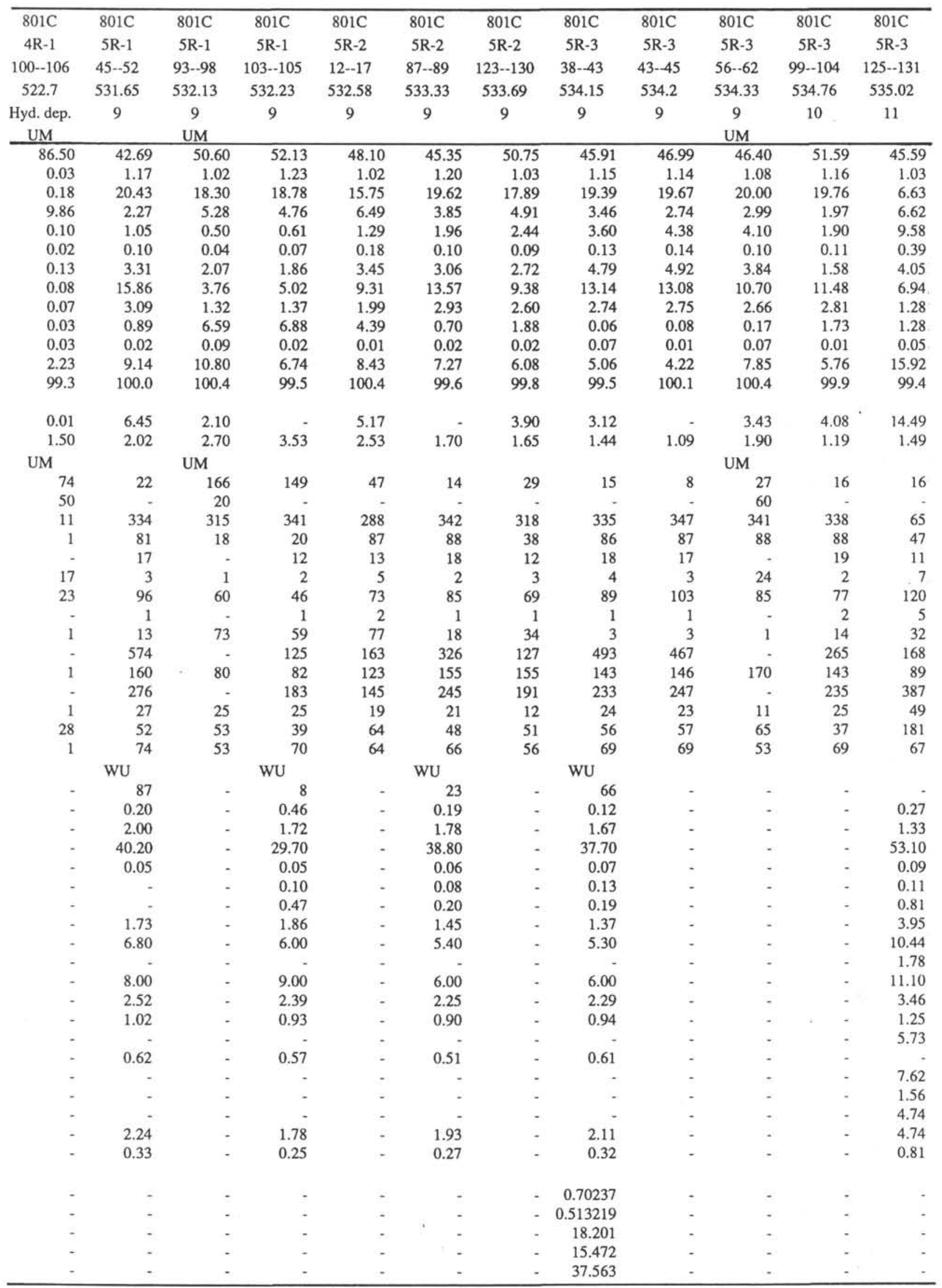


Table 1 (continued).

\begin{tabular}{|c|c|c|c|c|c|c|c|c|c|c|c|}
\hline $801 \mathrm{C}$ & $801 C$ & $801 C$ & $801 C$ & $801 C$ & $801 C$ & $801 C$ & $801 C$ & $801 \mathrm{C}$ & $801 \mathrm{C}$ & $801 C$ & $801 \mathrm{C}$ \\
\hline $5 R-4$ & $5 R-5$ & $5 R-5$ & $5 R-5$ & $6 \mathrm{R}-2$ & $6 \mathrm{R}-2$ & $6 \mathrm{R}-3$ & $6 \mathrm{R}-3$ & $6 R-3$ & $6 R_{-4}$ & $6 R-5$ & $6 R-5$ \\
\hline $65--70$ & $11--17$ & $65--69$ & 73--78 & $29--34$ & $119--124$ & $1--5$ & $37-42$ & $75--80$ & $49--54$ & $44--50$ & $52--57$ \\
\hline 535.92 & 536.8 & 537.34 & 537.42 & 542.08 & 542.98 & 543.2 & 543.56 & 543.94 & 545.14 & 546.47 & 546.55 \\
\hline \multirow[t]{2}{*}{13} & 13 & 13 & 13 & 16 & 16 & 18 & 20 & 20 & 20 & 21 & 21 \\
\hline & UM & & & & & UM & & & \multicolumn{3}{|c|}{ UM } \\
\hline 47.42 & 42.40 & 48.13 & 47.07 & 46.43 & 46.40 & 44.00 & 40.58 & 47.27 & 46.63 & 47.10 & 47.32 \\
\hline 0.97 & 0.85 & 0.96 & 0.93 & 0.98 & 1.00 & 1.00 & 0.98 & 0.97 & 0.89 & 1.75 & 1.85 \\
\hline 16.46 & 16.50 & 16.61 & 16.51 & 16.32 & 17.22 & 18.10 & 17.53 & 17.10 & 16.44 & 13.10 & 13.59 \\
\hline 2.37 & 1.80 & 9.07 & 3.36 & 2.54 & 3.44 & 4.61 & 2.87 & 3.04 & 2.19 & 2.99 & 2.78 \\
\hline 6.31 & 4.30 & 0.00 & 5.72 & 5.90 & 4.85 & 3.20 & 2.48 & 5.65 & 6.32 & 9.10 & 8.77 \\
\hline 0.14 & 0.15 & 0.14 & 0.17 & 0.14 & 0.16 & 0.14 & 0.18 & 0.14 & 0.14 & 0.21 & 0.19 \\
\hline 8.58 & 4.75 & 7.29 & 7.17 & 9.67 & 7.69 & 3.75 & 2.71 & 7.86 & 10.14 & 6.39 & 6.56 \\
\hline 12.08 & 17.70 & 12.83 & 12.88 & 11.48 & 12.84 & 10.20 & 16.37 & 12.54 & 12.29 & 11.60 & 11.66 \\
\hline 2.24 & 2.16 & 2.34 & 2.17 & 2.16 & 2.37 & 2.52 & 2.63 & 2.34 & 2.03 & 2.41 & 2.71 \\
\hline 0.08 & 0.09 & 0.09 & 0.05 & 0.05 & 0.02 & 0.37 & 0.90 & 0.02 & 0.04 & 0.19 & 0.10 \\
\hline 0.01 & 0.06 & 0.13 & 0.01 & 0.05 & 0.01 & 0.07 & 0.01 & 0.06 & 0.01 & 0.14 & 0.08 \\
\hline 3.18 & 9.31 & 2.79 & 3.93 & 4.45 & 3.91 & 12.00 & 12.28 & 3.32 & 2.87 & 3.62 & 3.60 \\
\hline 99.8 & 100.6 & 100.4 & 100.0 & 100.2 & 99.9 & 100.3 & 99.5 & 100.3 & 100.0 & 99.6 & 99.2 \\
\hline 2.14 & 7.52 & - & 2.59 & 2.58 & 2.61 & 5.19 & 10.03 & 1.67 & 1.92 & 2.87 & 2.77 \\
\hline 0.81 & 1.40 & - & 1.09 & 1.51 & 1.10 & 2.30 & 1.86 & 1.05 & 0.73 & 0.50 & 0.55 \\
\hline & UM & & & & & UM & & & & & \\
\hline 15 & 1 & 16 & 16 & 8 & 6 & 38 & 7 & 14 & 10 & 23 & 21 \\
\hline - & 65 & - & - & - & - & 52 & - & - & - & - & - \\
\hline 464 & 447 & 523 & 570 & 559 & 557 & 571 & 515 & 402 & 455 & 135 & 161 \\
\hline 81 & 74 & 75 & 81 & 79 & 82 & 85 & 85 & 88 & 77 & 71 & 73 \\
\hline 12 & - & 14 & 15 & 14 & 14 & - & 15 & 16 & 14 & - & 20 \\
\hline 3 & 14 & 4 & 3 & 3 & 3 & 20 & 4 & 3 & 4 & 18 & 7 \\
\hline 217 & 241 & 290 & 375 & 240 & 283 & 161 & 220 & 183 & 231 & 66 & 77 \\
\hline 1 & - & 2 & 1 & 1 & 1 & - & 1 & 2 & 1 & - & 3 \\
\hline 3 & 1 & 5 & 2 & 4 & 2 & 14 & 9 & 1 & 2 & 11 & 3 \\
\hline 390 & - & 387 & 797 & 383 & 505 & - & 323 & 399 & 389 & - & 525 \\
\hline 113 & 168 & 155 & 116 & 100 & 109 & 131 & 130 & 103 & 138 & 93 & 131 \\
\hline 199 & - & 208 & 198 & 217 & 213 & - & 264 & 211 & 186 & - & 400 \\
\hline 26 & 15 & 22 & 23 & 24 & 26 & 27 & 18 & 25 & 22 & 27 & 42 \\
\hline 62 & 59 & 64 & 65 & 53 & 69 & 62 & 52 & 63 & 56 & 99 & 93 \\
\hline 56 & 41 & 56 & 55 & 55 & 60 & 55 & 48 & 58 & 52 & 103 & 114 \\
\hline
\end{tabular}

WU

$\begin{array}{rrrr}48 & - & - & - \\ 0.11 & 0.05 & - & 0.07 \\ 1.42 & 1.50 & - & 1.18 \\ 39.50 & 40.40 & - & 26.70 \\ 0.06 & 0.02 & - & 0.04 \\ 0.08 & 0.03 & - & 0.03 \\ 0.20 & 0.02 & - & 0.67 \\ 0.99 & 1.51 & - & 1.83 \\ 3.90 & 5.23 & - & 5.19 \\ - & 1.26 & - & 1.05 \\ 5.00 & 7.04 & - & 5.69 \\ 1.97 & 1.92 & - & 1.54 \\ 0.80 & 0.87 & - & 0.70 \\ - & 3.37 & - & 2.46 \\ 0.56 & - & - & - \\ - & 4.06 & - & 2.89 \\ - & 0.82 & - & 0.58 \\ - & 2.53 & - & 1.82 \\ 2.29 & 2.44 & - & 1.72 \\ 0.35 & 0.44 & - & 0.30\end{array}$


Table 1 (continued).

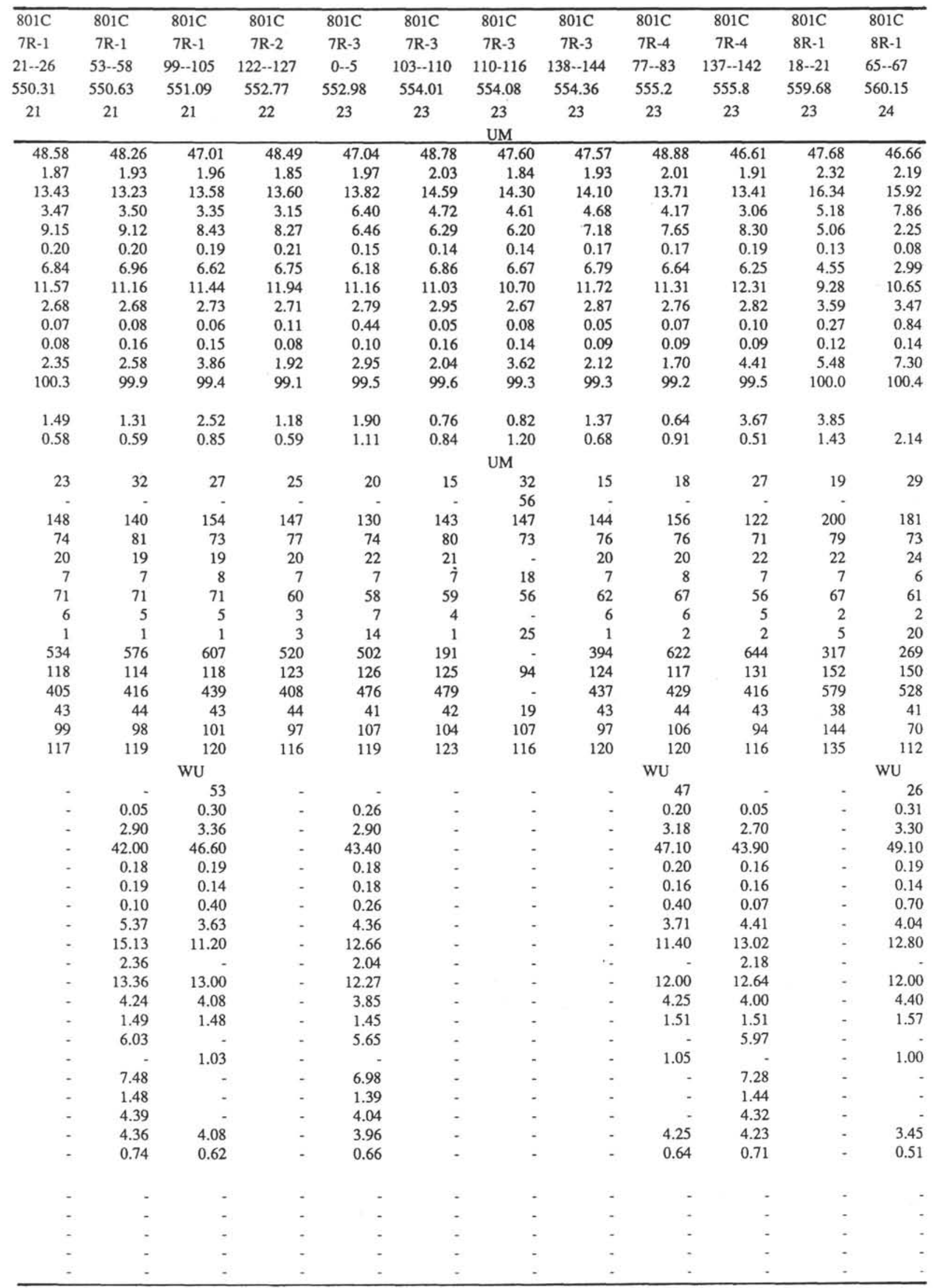


Table 1 (continued).

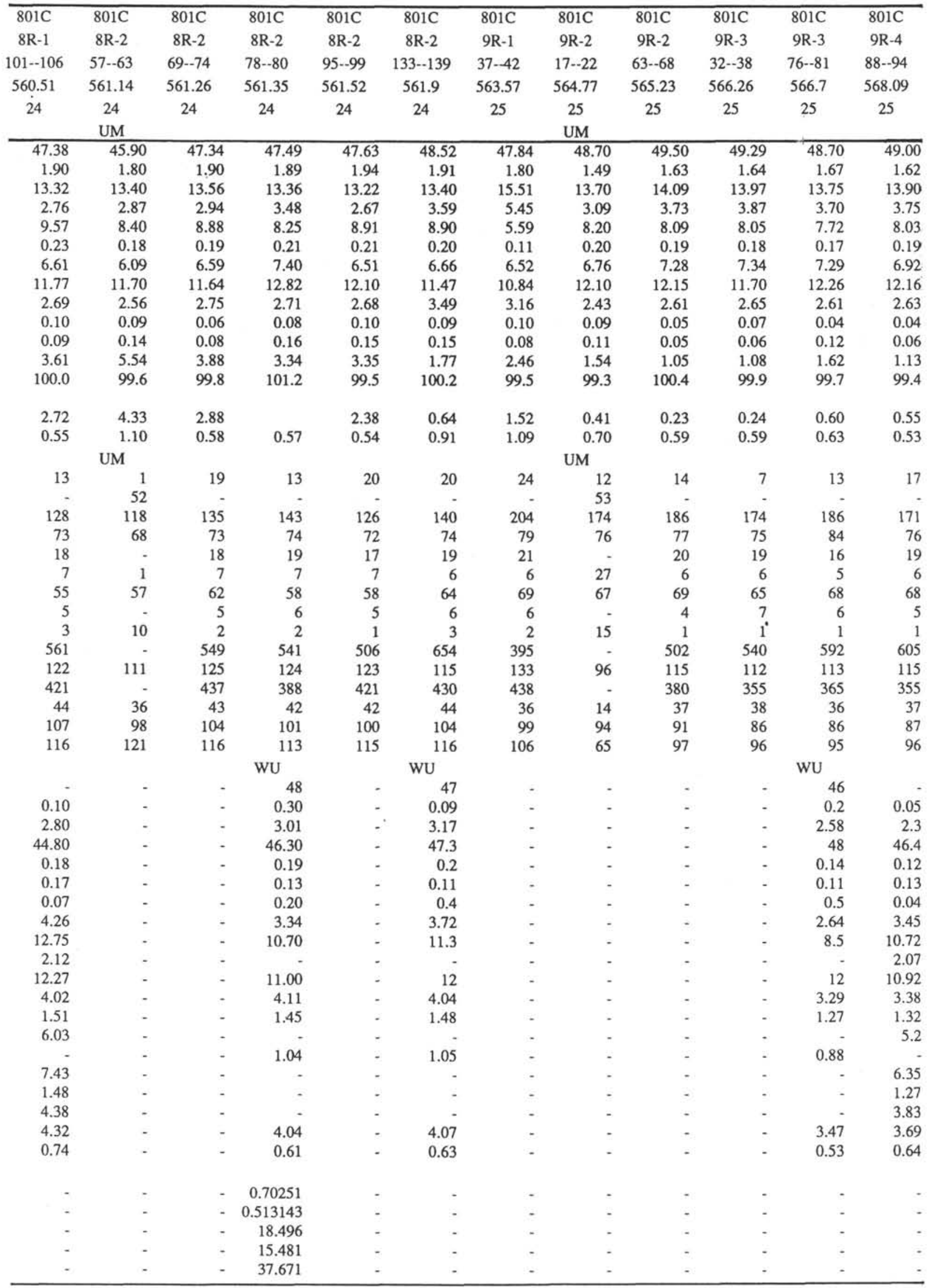


Table 1 (continued).

\begin{tabular}{|c|c|c|c|c|c|c|c|c|c|c|c|}
\hline $801 C$ & $801 C$ & $801 C$ & $801 \mathrm{C}$ & $801 C$ & $801 C$ & $801 C$ & $801 C$ & $801 C$ & $801 C$ & $801 C$ & $801 C$ \\
\hline 9R-5 & 9R-5 & $10 \mathrm{R}-1$ & $10 \mathrm{R}-2$ & $10 \mathrm{R}-2$ & $10 \mathrm{R}-2$ & $10 \mathrm{R}-4$ & $10 R-5$ & $10 \mathrm{R}-5$ & 10R-5 & $10 \mathrm{R}-6$ & $10 \mathrm{R}-6$ \\
\hline $0--7$ & $20--24$ & $55--60$ & $25--31$ & 93--97 & $121--127$ & $64--70$ & $53--58$ & $62--67$ & 99--104 & $21-26$ & $47--52$ \\
\hline 568.61 & 568.81 & 569.55 & 570.75 & 571.43 & 573.13 & 574.06 & 575.34 & 575.43 & 575.8 & 576.37 & 576.63 \\
\hline 25 & 25 & 25 & 25 & $\begin{array}{c}25 \\
\text { UM }\end{array}$ & 25 & 25 & 25 & 25 & 25 & 25 & 25 \\
\hline 49.40 & 49.10 & 49.44 & 49.60 & 49.00 & 48.92 & 48.73 & 49.42 & 49.46 & 49.73 & 48.77 & 48.73 \\
\hline 1.63 & 1.71 & 1.62 & 1.63 & 1.50 & 1.59 & 1.57 & 1.59 & 1.62 & 1.63 & 1.64 & 1.61 \\
\hline 13.95 & 14.05 & 14.02 & 14.01 & 13.60 & 14.03 & 13.92 & 13.90 & 14.03 & 13.98 & 14.12 & 14.07 \\
\hline 3.92 & 3.49 & 3.82 & 3.82 & 3.03 & 3.72 & 3.06 & 12.83 & 3.88 & 3.77 & 11.95 & 3.76 \\
\hline 8.15 & 7.81 & 8.15 & 8.29 & 8.70 & 7.88 & 8.56 & 0.00 & 7.98 & 8.12 & 0.00 & 7.44 \\
\hline 0.18 & 0.18 & 0.18 & 0.19 & 0.20 & 0.19 & 0.20 & 0.19 & 0.18 & 0.18 & 0.18 & 0.19 \\
\hline 7.44 & 7.19 & 7.28 & 7.33 & 7.16 & 7.59 & 7.06 & 7.18 & 7.09 & 7.25 & 6.99 & 7.14 \\
\hline 11.83 & 11.73 & 11.95 & 11.74 & 11.70 & 12.04 & 12.47 & 12.12 & 12.01 & 11.83 & 12.66 & 12.58 \\
\hline 2.54 & 2.62 & 2.56 & 2.67 & 2.48 & 2.59 & 2.53 & 2.59 & 2.62 & 2.62 & 2.73 & 2.78 \\
\hline 0.05 & 0.05 & 0.06 & 0.06 & 0.05 & 0.03 & 0.04 & 0.07 & 0.06 & 0.06 & 0.07 & 0.08 \\
\hline 0.06 & 0.06 & 0.06 & 0.06 & 0.11 & 0.05 & 0.05 & 0.20 & 0.05 & 0.13 & 0.20 & 0.06 \\
\hline 0.99 & 1.31 & 1.08 & 1.04 & 1.16 & 1.04 & 1.25 & 0.28 & 1.01 & 1.16 & 0.66 & 1.72 \\
\hline 100.1 & 99.3 & 100.2 & 100.4 & 99.7 & 99.7 & 99.4 & 100.4 & 100.0 & 100.5 & 100.0 & 100.2 \\
\hline 0.16 & 0.20 & 0.28 & 0.14 & 0.14 & 0.13 & 0.54 & - & 0.23 & 0.08 & - & 1.05 \\
\hline 0.61 & 0.97 & 0.55 & 0.57 & 0.80 & 0.66 & 0.54 & - & 0.58 & 0.59 & - & 0.55 \\
\hline \multicolumn{12}{|c|}{ UM } \\
\hline 18 & 16 & 14 & 24 & 37 & 21 & 16 & 14 & 14 & 9 & 18 & 25 \\
\hline - & - & - & - & 51 & - & - & - & - & - & - & 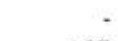 \\
\hline 175 & 203 & 175 & 171 & 168 & 199 & 205 & 200 & 187 & 193 & 199 & 187 \\
\hline 74 & 78 & 75 & 75 & 80 & 76 & 75 & 74 & 75 & 76 & 76 & 74 \\
\hline 17 & 21 & 19 & 19 & - & 20 & 19 & 16 & 19 & 21 & 16 & 17 \\
\hline 6 & 5 & 7 & 6 & 24 & 6 & 6 & 5 & 4 & 6 & 5 & 6 \\
\hline 66 & 69 & 70 & 65 & 67 & 72 & 70 & 67 & 71 & 68 & 70 & 69 \\
\hline 7 & 6 & 6 & 8 & - & 8 & 6 & 5 & 3 & 7 & 6 & 4 \\
\hline 1 & 1 & 1 & 1 & 1 & 2 & 1 & 4 & 1 & 1 & 3 & 3 \\
\hline 579 & 670 & 602 & 595 & - & 565 & 617 & 487 & 571 & 576 & 516 & 431 \\
\hline 114 & 108 & 113 & 110 & 103 & 113 & 115 & 112 & 112 & 108 & 120 & 125 \\
\hline 359 & 368 & 365 & 360 & . & 363 & 357 & 347 & 355 & 358 & 349 & 371 \\
\hline 37 & 37 & 38 & 37 & 30 & 37 & 37 & 36 & 38 & 38 & 37 & 36 \\
\hline 84 & 93 & 85 & 91 & 91 & 89 & 84 & 80 & 83 & 83 & 86 & 90 \\
\hline 97 & 95 & 97 & 95 & 90 & 94 & 95 & 93 & 96 & 95 & 96 & 96 \\
\hline
\end{tabular}

WU

$\begin{array}{rr}46 & - \\ 0.17 & 0.05 \\ 2.55 & 2.4 \\ 46.3 & 45 \\ 0.13 & 0.13 \\ 0.11 & 0.12 \\ 0.3 & 0.05 \\ 2.75 & 3.48 \\ 8.8 & 10.78 \\ - & 2.06 \\ 7 & 10.65 \\ 3.38 & 3.44 \\ 1.28 & 1.33 \\ - & 5.2 \\ 0.94 & 0 \\ - & 6.38 \\ - & 1.27 \\ - & 3.83 \\ 3.53 & 3.69 \\ 0.53 & 0.62\end{array}$


Table 1 (continued).

\begin{tabular}{|c|c|c|c|c|c|c|c|c|c|c|c|}
\hline $801 \mathrm{C}$ & $801 C$ & $801 \mathrm{C}$ & $801 C$ & $801 \mathrm{C}$ & $801 C$ & $801 \mathrm{C}$ & $801 C$ & $801 C$ & $801 C$ & $801 C$ & $801 C$ \\
\hline $10 \mathrm{R}-6$ & $10 \mathrm{R}-6$ & $11 \mathrm{R}-1$ & $11 \mathrm{R}-1$ & $11 \mathrm{R}-2$ & $11 R-2$ & $11 \mathrm{R}-3$ & $11 \mathrm{R}-3$ & $11 R-3$ & $12 \mathrm{R}-1$ & $12 \mathrm{R}-1$ & $12 \mathrm{R}-2$ \\
\hline $67--70$ & $125-131$ & $18--23$ & $121-126$ & $81--86$ & $131--136$ & $14--18$ & $78--83$ & $121-126$ & $12--14$ & $101-104$ & $44-149$ \\
\hline 576.83 & 577.41 & 578.08 & 579.11 & 580.11 & 580.16 & 580.94 & 581.58 & 582.01 & 587.42 & 588.31 & 589.24 \\
\hline \multirow[t]{2}{*}{25} & 26 & 27 & 27 & 27 & 27 & 28 & 29 & 29 & Interpillow & 31 & 32 \\
\hline & UM & & & & & & UM & & UM & & \\
\hline 48.64 & 46.90 & 47.99 & 48.45 & 49.02 & 47.19 & 48.90 & 46.50 & 46.01 & 36.00 & 48.27 & 49.37 \\
\hline 1.78 & 0.84 & 2.30 & 2.19 & 2.18 & 2.11 & 1.95 & 1.72 & 1.95 & 0.40 & 2.39 & 2.51 \\
\hline 14.55 & 15.80 & 14.61 & 14.18 & 13.66 & 15.15 & 14.44 & 14.10 & 14.52 & 19.10 & 14.66 & 14.90 \\
\hline 3.95 & 3.29 & 5.66 & 5.04 & 13.84 & 5.19 & 3.86 & 5.15 & 4.13 & 5.67 & 6.35 & 5.80 \\
\hline 6.14 & 5.50 & 6.80 & 7.26 & 0.00 & 6.64 & 8.07 & 5.80 & 8.24 & 1.80 & 6.65 & 5.90 \\
\hline 0.16 & 0.15 & 0.16 & 0.19 & 0.21 & 0.16 & 0.19 & 0.16 & 0.19 & 0.40 & 0.19 & 0.17 \\
\hline 7.12 & 6.60 & 6.77 & 6.80 & 6.63 & 6.00 & 5.83 & 5.29 & 5.26 & 4.42 & 6.55 & 6.45 \\
\hline 12.26 & 14.60 & 10.86 & 11.86 & 11.27 & 12.01 & 12.02 & 12.10 & 12.54 & 22.80 & 10.15 & 9.92 \\
\hline 2.77 & 1.88 & 2.90 & 2.81 & 2.78 & 2.59 & 2.51 & 2.42 & 2.57 & 0.44 & 2.98 & 3.28 \\
\hline 0.07 & 0.04 & 0.07 & 0.06 & 0.07 & 0.10 & 0.11 & 0.35 & 0.09 & 1.44 & 0.26 & 0.06 \\
\hline 0.06 & 0.06 & 0.15 & 0.11 & 0.12 & 0.17 & 0.10 & 0.13 & 0.10 & 0.02 & 0.14 & 0.14 \\
\hline 2.19 & 3.47 & 1.44 & 1.34 & 0.28 & 2.29 & 1.69 & 5.93 & 4.60 & 24.30 & 1.36 & 1.44 \\
\hline 99.7 & 99.7 & 99.7 & 100.3 & 100.1 & 99.6 & 99.7 & 100.3 & 100.2 & 117.0 & 100.0 & 99.9 \\
\hline 1.00 & 1.75 & 0.54 & 0.53 & 0.43 & 0.81 & 0.67 & 3.21 & 3.44 & 2.57 & 0.33 & 0.49 \\
\hline \multirow[t]{2}{*}{1.03} & 1.10 & 0.91 & 0.71 & 0.56 & 1.32 & 0.81 & 1.40 & 0.86 & 2.00 & 1.08 & 1.04 \\
\hline & UM & & & & & & UM & & UM & & \\
\hline 9 & 10 & 25 & 26 & 27 & 16 & 2 & 15 & 32 & 1 & 11 & 22 \\
\hline- & - & - & - & - & - & - & 34 & - & 11 & - & - \\
\hline 200 & 377 & 199 & 188 & 170 & 318 & 292 & 257 & 315 & 10 & 122 & 101 \\
\hline 78 & 93 & 72 & 71 & 69 & 69 & 68 & 48 & 66 & 42 & 76 & 70 \\
\hline 19 & - & 21 & 21 & 20 & 18 & 21 & - & 18 & $\cdot$ & 21 & 23 \\
\hline 6 & 1 & 7 & 9 & 8 & 7 & 6 & 10 & 7 & 1 & 8 & 8 \\
\hline 67 & 113 & 66 & 66 & 64 & 88 & 86 & 47 & 67 & 17 & 58 & 56 \\
\hline 3 & - & 6 & 6 & 8 & 6 & 7 & - & 4 & - & 5 & 5 \\
\hline 1 & 1 & 1 & 1 & 1 & 4 & 2 & 18 & 3 & 50 & 6 & 1 \\
\hline 275 & - & 323 & 249 & 608 & 490 & 441 & - & 443 & - & 430 & 331 \\
\hline 118 & 200 & 140 & 137 & 129 & 112 & 110 & 108 & 113 & 46 & 134 & 142 \\
\hline 408 & - & 488 & 439 & 404 & 441 & 386 & - & 401 & - & 518 & 551 \\
\hline 38 & 1 & 46 & 44 & 45 & 44 & 44 & 23 & 45 & 15 & 51 & 58 \\
\hline 95 & 68 & 109 & 106 & 101 & 111 & 103 & 84 & 111 & 29 & 124 & 109 \\
\hline \multirow[t]{2}{*}{101} & 51 & 149 & 141 & 138 & 126 & 124 & 111 & 122 & 18 & 153 & 161 \\
\hline & & & & & WU & & & & & & \\
\hline 47 & - & - & - & - & 48 & - & - & - & - & - & - \\
\hline 0.10 & - & - & 0.10 & - & 0.40 & - & - & 0.10 & - & - & - \\
\hline 2.60 & - & - & 3.50 & - & 3.19 & - & - & 3.20 & - & - & - \\
\hline 45.90 & - & - & 44.20 & - & 44.10 & - & - & 42.00 & - & - & - \\
\hline 0.12 & - & - & 0.24 & - & 0.16 & - & - & 0.15 & - & - & - \\
\hline 0.11 & - & - & 0.25 & - & 0.07 & - & - & 0.13 & - & - & - \\
\hline 0.10 & - & - & 0.08 & - & 0.60 & - & - & 0.08 & - & - & - \\
\hline 3.26 & - & - & 4.94 & - & 3.34 & - & - & 3.87 & - & - & - \\
\hline 10.08 & - & - & 14.71 & - & 10.80 & - & - & 12.28 & - & - & - \\
\hline 1.78 & - & - & 2.48 & - & - & - & - & 1.99 & - & - & - \\
\hline 10.38 & - & - & 13.45 & - & 10.00 & - & - & 12.00 & - & - & - \\
\hline 3.33 & - & - & 4.30 & - & 4.25 & - & - & 3.96 & - & - & - \\
\hline 1.30 & - & - & 1.62 & - & 1.46 & - & - & 1.48 & - & - & - \\
\hline 5.01 & - & - & 6.42 & - & - & - & - & 5.95 & - & - & - \\
\hline - & $\cdot$ & - & - & - & 1.05 & - & - & - & - & - & $\cdot$ \\
\hline 6.24 & - & - & 7.67 & - & - & - & - & 7.43 & - & - & $\cdot$ \\
\hline 1.24 & - & - & 1.52 & - & - & - & - & 1.48 & - & - & - \\
\hline 3.71 & - & - & 4.51 & - & - & - & - & 4.33 & - & - & - \\
\hline 3.68 & - & - & 4.46 & - & 4.08 & - & - & 4.30 & - & - & $\cdot$ \\
\hline 0.62 & - & - & 0.73 & - & 0.61 & - & - & 0.73 & $\cdot$ & - & - \\
\hline 0.70239 & - & - & - & - & 0.70248 & - & - & - & - & - & - \\
\hline 0.513027 & - & - & - & - & 0.513001 & - & - & - & - & - & - \\
\hline 18.769 & - & - & - & - & 18.326 & - & - & - & - & - & $\cdot$ \\
\hline 15.543 & - & - & - & - & 15.492 & - & - & - & - & - & - \\
\hline 37.857 & - & - & - & - & 37.676 & - & - & - & - & - & - \\
\hline
\end{tabular}


Table 1 (continued).

\begin{tabular}{|c|c|c|c|c|c|c|c|c|c|c|c|}
\hline $801 C$ & $801 C$ & $801 C$ & $801 \mathrm{C}$ & $802 \mathrm{~A}$ & $802 \mathrm{~A}$ & $802 \mathrm{~A}$ & $802 \mathrm{~A}$ & $802 \mathrm{~A}$ & $802 \mathrm{~A}$ & $802 \mathrm{~A}$ & $802 \mathrm{~A}$ \\
\hline $12 R-2$ & $12 \mathrm{R}-3$ & $12 \mathrm{R}-3$ & $12 \mathrm{R}-3$ & $57 R--2$ & $57 \mathrm{R}-3$ & $57 \mathrm{R}-3$ & $58 \mathrm{R}-1$ & $58 \mathrm{R}-1$ & $58 \mathrm{R}-1$ & $58 \mathrm{R}-2$ & $58 \mathrm{R}-2$ \\
\hline $103-109$ & $32--37$ & $57--62$ & 86--90 & $109--115$ & $1-6$ & $37-42$ & $9-13$ & $130--135$ & $96--100$ & $18--23$ & $91--96$ \\
\hline 589.83 & 590.58 & 590.83 & 591.12 & 509.11 & 509.61 & 509.97 & 516.09 & 517.3 & 517.68 & 517.68 & 518.41 \\
\hline 32 & 32 & 32 & 32 & 1 & 1 & 1 & 2 & 2 & 2 & 2 & 2 \\
\hline & & & & & \multicolumn{7}{|c|}{ UM } \\
\hline 48.55 & 48.42 & 48.90 & 48.44 & 48.66 & 49.50 & 49.96 & 50.56 & 49.60 & 49.55 & 50.76 & 49.18 \\
\hline 2.26 & 2.30 & 2.29 & 2.16 & 1.42 & 1.17 & 1.18 & 1.13 & 0.99 & 1.09 & 1.11 & 1.19 \\
\hline 13.26 & 13.25 & 13.58 & 13.53 & 16.53 & 14.80 & 14.50 & 14.45 & 13.50 & 14.21 & 14.20 & 14.27 \\
\hline 4.15 & 3.63 & 4.61 & 4.45 & 5.91 & 4.10 & 3.56 & 3.75 & 5.42 & 3.07 & 3.31 & 3.46 \\
\hline 8.91 & 8.78 & 7.96 & 8.66 & 4.75 & 6.43 & 7.26 & 7.33 & 7.00 & 8.58 & 8.29 & 8.02 \\
\hline 0.23 & 0.22 & 0.22 & 0.24 & 0.16 & 0.20 & 0.21 & 0.19 & 0.19 & 0.21 & 0.18 & 0.20 \\
\hline 6.55 & 6.80 & 6.83 & 6.78 & 7.42 & 7.87 & 7.79 & 7.68 & 6.65 & 8.05 & 7.50 & 7.69 \\
\hline 11.29 & 11.30 & 11.11 & 11.47 & 8.53 & 11.70 & 11.69 & 11.72 & 11.20 & 11.76 & 11.69 & 11.73 \\
\hline 2.80 & 2.79 & 2.82 & 2.68 & 2.78 & 2.65 & 2.57 & 2.10 & 1.97 & 2.00 & 2.01 & 2.10 \\
\hline 0.09 & 0.04 & 0.08 & 0.04 & 0.84 & 0.10 & 0.09 & 0.08 & 0.70 & 0.07 & 0.10 & 0.24 \\
\hline 0.12 & 0.19 & 0.12 & 0.12 & 0.10 & 0.04 & 0.03 & 0.03 & 0.08 & 0.03 & 0.03 & 0.03 \\
\hline 1.38 & 1.22 & 1.07 & 1.11 & 2.70 & 1.17 & 1.02 & 1.00 & 1.39 & 0.98 & 0.97 & 1.00 \\
\hline 99.6 & 98.9 & 99.6 & 99.7 & 99.8 & 99.7 & 99.9 & 100.0 & 99.5 & 99.6 & 100.2 & 99.1 \\
\hline 0.28 & 0.27 & 0.10 & 0.38 & 0.58 & 0.12 & 0.22 & 0.18 & 0.19 & 0.10 & 0.10 & 0.16 \\
\hline \multirow[t]{2}{*}{0.93} & 0.51 & 0.79 & 0.55 & 2.05 & 1.11 & 0.63 & 0.57 & 0.90 & 0.54 & 0.44 & 0.64 \\
\hline & & & & & \multicolumn{7}{|c|}{ UM } \\
\hline 24 & 22 & 25 & 17 & 56 & 10 & 26 & 9 & 24 & 16 & 32 & 11 \\
\hline - & - & - & - & - & - & - & - & 46 & - & - & - \\
\hline 106 & 93 & 101 & 106 & 167 & 156 & 138 & 125 & 120 & 150 & 131 & 151 \\
\hline 71 & 74 & 73 & 71 & 162 & 157 & 155 & 153 & 63 & 153 & 159 & 148 \\
\hline 18 & 22 & 21 & 22 & 20 & 18 & 17 & 16 & - & 17 & 21 & 16 \\
\hline 7 & 7 & 7 & 7 & 9 & 4 & 4 & 5 & $<10$ & 5 & 4 & 4 \\
\hline 59 & 56 & 60 & 57 & 88 & 103 & 100 & 96 & 77 & 98 & 95 & 96 \\
\hline 6 & 5 & 6 & 8 & 4 & 5 & 6 & 6 & - & 6 & 6 & 6 \\
\hline 3 & 1 & 2 & 1 & 8 & 4 & 2 & 1 & 30 & 2 & 1 & 6 \\
\hline 563 & 574 & 623 & 569 & 234 & 400 & 275 & 189 & - & 252 & 302 & 279 \\
\hline 121 & 121 & 119 & 122 & 161 & 98 & 104 & 102 & 77 & 99 & 97 & 98 \\
\hline 427 & 437 & 458 & 432 & 423 & 357 & 354 & 359 & - & 332 & 342 & 346 \\
\hline 49 & 49 & 49 & 49 & 28 & 25 & 24 & 25 & 10 & 24 & 25 & 26 \\
\hline 110 & 101 & 108 & 104 & 119 & 92 & 90 & 91 & 83 & 95 & 91 & 94 \\
\hline \multirow[t]{2}{*}{143} & 143 & 144 & 141 & 101 & 64 & 67 & 66 & 48 & 65 & 64 & 65 \\
\hline & wU & wU & & & & WU & & & & & \\
\hline - & 47 & 47 & - & & - & 52 & & - & - & - & - \\
\hline - & 0.20 & 0.30 & - & 0.09 & - & 0.20 & 0.10 & - & - & 0.01 & . \\
\hline - & 3.75 & 3.83 & - & 2.30 & - & 1.76 & 1.90 & - & - & 1.60 & - \\
\hline - & 47.30 & 46.80 & - & 48.80 & - & 49.40 & 46.50 & - & - & 47.80 & - \\
\hline - & 0.22 & 0.23 & - & 0.46 & - & 0.19 & 0.19 & - & - & 0.17 & - \\
\hline - & 0.16 & 0.14 & - & 0.62 & - & 0.22 & 0.28 & - & - & 0.26 & . \\
\hline - & 0.60 & 0.40 & - & 0.17 & - & 0.40 & 0.08 & . & . & 0.09 & - \\
\hline$\cdot$ & 4.33 & 4.31 & - & 9.07 & - & 3.17 & 3.93 & - & - & 3.83 & . \\
\hline$\cdot$ & 14.30 & 13.20 & - & 19.88 & - & 8.60 & 9.93 & - & - & 10.02 & - \\
\hline - & - & - & - & 2.65 & - & 0.00 & 1.46 & - & - & 1.46 & - \\
\hline- & 19.00 & 14.00 & - & 12.73 & - & 15.00 & 7.94 & - & - & 7.76 & - \\
\hline - & 4.71 & 4.83 & - & 3.19 & - & 2.32 & 2.25 & - & - & 2.23 & . \\
\hline- & 1.66 & 1.65 & - & 1.22 & - & 0.88 & 0.89 & - & . & 0.87 & . \\
\hline- & - & - & - & 4.16 & - & - & 3.34 & - & - & 3.28 & - \\
\hline - & 1.24 & 1.24 & - & - & - & 0.57 & - & - & - & - & . \\
\hline- & - & - & - & 4.61 & - & - & 4.08 & . & . & 4.08 & . \\
\hline - & - & - & - & 0.87 & - & - & 0.82 & - & - & 0.83 & . \\
\hline - & - & - & - & 2.52 & - & - & 2.47 & - & - & 2.44 & . \\
\hline - & 4.72 & 4.71 & . & 2.36 & - & 2.37 & 2.49 & . & . & 2.38 & . \\
\hline - & 0.70 & 0.70 & - & 0.39 & - & 0.36 & 0.43 & - & - & 0.41 & . \\
\hline - & 0.70248 & - & - & - & - & - & - & - & - & - & . \\
\hline - & 0.512975 & - & - & - & . & - & - & - & - & - & . \\
\hline - & 19.123 & - & - & - & - & - & - & - & - & - & . \\
\hline - & 15.600 & - & - & - & - & - & - & - & - & - & . \\
\hline - & 38.181 & - & - & - & - & - & - & - & - & - & . \\
\hline
\end{tabular}


Table 1 (continued).

\begin{tabular}{|c|c|c|c|c|c|c|c|c|c|c|c|}
\hline $802 \mathrm{~A}$ & $802 \mathrm{~A}$ & $802 \mathrm{~A}$ & $802 \mathrm{~A}$ & $802 \mathrm{~A}$ & $802 \mathrm{~A}$ & $802 \mathrm{~A}$ & $802 \mathrm{~A}$ & $802 \mathrm{~A}$ & $802 \mathrm{~A}$ & $802 \mathrm{~A}$ & $802 \mathrm{~A}$ \\
\hline $58 \mathrm{R}-2$ & $58 \mathrm{R}-2$ & $58 \mathrm{R}-2$ & $58 \mathrm{R}-2$ & $58 \mathrm{R}-3$ & $58 \mathrm{R}-3$ & $58 \mathrm{R}-3$ & $58 \mathrm{R}-3$ & $\mathrm{R}$ & $58 \mathrm{R}-4$ & 59R-1 & $59 \mathrm{R}-1$ \\
\hline $99--102$ & $110--115$ & $117--122$ & $142--149$ & $0-1$ & $23--28$ & 90--95 & $134--140$ & $24--29$ & $65--70$ & $7--12$ & $41-45$ \\
\hline 518.49 & 518.66 & 518.67 & 518.92 & 519 & 519.23 & 519.9 & 520.34 & 520.74 & 521.15 & 526.18 & 525.81 \\
\hline \multirow[t]{2}{*}{3} & 3 & 4 & 4 & 5 & 5 & 5 & 5 & 5 & 5 & 7 & 7 \\
\hline & & & & & & & & & & & UM \\
\hline 49.40 & - & 49.36 & - & - & 50.46 & 49.34 & 50.66 & 50.64 & 49.32 & 49.46 & 49.40 \\
\hline 1.22 & - & 1.15 & - & - & 1.22 & 1.10 & 1.14 & 1.17 & 1.12 & 1.21 & 1.01 \\
\hline 14.39 & - & 14.38 & - & - & 14.78 & 14.25 & 14.35 & 14.10 & 14.55 & 14.72 & 13.70 \\
\hline 2.54 & - & 3.75 & - & - & 3.69 & 4.40 & 3.43 & 3.01 & 3.87 & 3.60 & 2.73 \\
\hline 8.83 & - & 8.17 & - & - & 6.36 & 7.56 & 7.28 & 8.35 & 8.23 & 7.63 & 8.70 \\
\hline 0.21 & - & 0.21 & - & - & 0.16 & 0.20 & 0.17 & 0.17 & 0.21 & 0.19 & 0.21 \\
\hline 7.66 & - & 7.68 & - & - & 7.34 & 7.71 & 8.03 & 7.56 & 7.57 & 7.98 & 7.58 \\
\hline 11.78 & - & 11.87 & - & - & 11.96 & 11.73 & 11.93 & 11.65 & 11.96 & 11.97 & 12.00 \\
\hline 1.95 & - & 1.95 & - & - & 2.33 & 2.04 & 1.99 & 2.06 & 2.04 & 2.08 & 1.84 \\
\hline 0.18 & - & 0.14 & - & - & 0.08 & 0.25 & 0.03 & 0.08 & 0.13 & 0.08 & 0.11 \\
\hline 0.02 & - & 0.03 & - & - & 0.02 & 0.02 & 0.10 & 0.02 & 0.03 & 0.03 & 0.08 \\
\hline 0.86 & - & 1.08 & - & - & 1.50 & 1.09 & 0.99 & 0.95 & 1.05 & 0.98 & 1.16 \\
\hline 99.0 & - & 99.8 & - & - & 99.9 & 99.7 & 100.1 & 99.8 & 100.1 & 99.9 & 99.5 \\
\hline 0.11 & - & 0.13 & - & - & 0.62 & 0.15 & 0.06 & 0.10 & 0.08 & 0.13 & 0.16 \\
\hline \multirow[t]{2}{*}{0.54} & - & 0.90 & - & - & 0.65 & 0.65 & 0.51 & 0.46 & 0.94 & 0.64 & 0.60 \\
\hline & & & & & & & & & & & UM \\
\hline 20 & - & 14 & - & - & 18 & 12 & 28 & 13 & 22 & 34 & 30 \\
\hline- & - & - & - & - & - & - & - & - & - & - & 55 \\
\hline 155 & - & 159 & - & - & 134 & 141 & 136 & 136 & 180 & 144 & 125 \\
\hline 157 & - & 150 & - & - & 156 & 144 & 161 & 154 & 156 & 155 & 162 \\
\hline 15 & - & 16 & - & - & 16 & 19 & 16 & 18 & 17 & 14 & - \\
\hline 4 & - & 4 & - & - & 5 & 4 & 3 & 4 & 4 & 5 & 18 \\
\hline 99 & - & 101 & - & - & 100 & 99 & 99 & 93 & 110 & 93 & 95 \\
\hline 4 & - & 5 & - & - & 4 & 6 & 2 & 7 & 4 & 4 & - \\
\hline 2 & - & 4 & - & . & 1 & 8 & 1 & 1 & 1 & 1 & $<10$ \\
\hline 284 & - & 322 & - & - & 217 & 272 & 242 & 315 & 330 & 249 & - \\
\hline 95 & - & 94 & - & - & 106 & 99 & 99 & 96 & 95 & 98 & 99 \\
\hline 358 & - & 355 & - & - & 349 & 351 & 363 & 340 & 367 & 344 & - \\
\hline 25 & - & 25 & - & - & 25 & 24 & 24 & 24 & 25 & 25 & 19 \\
\hline 97 & - & 94 & - & - & 93 & 92 & 94 & 90 & 97 & 88 & 90 \\
\hline \multirow[t]{2}{*}{65} & - & 65 & - & - & 65 & 63 & 63 & 63 & 65 & 65 & 58 \\
\hline & WU & WU & WU & WU & & WU & & & WU & & \\
\hline - & 44 & 47.6 & 47.8 & 47 & - & 50.1 & - & - & 48.4 & 54.8 & - \\
\hline- & 0.26 & - & - & - & 0.05 & 0.19 & - & - & 0.3 & 0.1 & - \\
\hline- & 1.66 & 1.76 & 1.83 & 1.79 & 1.8 & 1.64 & - & - & 1.83 & 1.5 & - \\
\hline- & 47.90 & 49 & 49.2 & 48.8 & 48.6 & 47.7 & - & - & 49 & 47.6 & - \\
\hline- & 0.15 & 0.17 & 0.18 & 0.18 & 0.16 & 0.17 & - & - & 0.17 & 0.17 & - \\
\hline - & 0.19 & 0.25 & 0.18 & 0.17 & 0.26 & 0.18 & - & - & 0.23 & 0.24 & - \\
\hline- & - & - & - & - & 0.1 & 0.5 & - & - & 0.3 & 0.11 & - \\
\hline- & 3.01 & 3.08 & 3.16 & 3.09 & 4.08 & 3 & - & - & 3.18 & 3.96 & - \\
\hline- & 7.70 & 8.5 & 8.2 & 7.9 & 10.31 & 8 & - & - & 8.3 & 10.28 & - \\
\hline- & - & - & - & - & 1.51 & - & - & - & - & 1.47 & - \\
\hline- & - & - & - & - & 8.21 & 6 & - & - & 9 & 8.3 & - \\
\hline- & 2.19 & 2.22 & 2.32 & 2.31 & 2.38 & 2.25 & - & - & - & 2.35 & - \\
\hline - & 0.81 & 0.87 & 0.85 & 0.86 & 0.91 & 0.81 & - & - & - & 0.91 & - \\
\hline - & - & - & - & - & 3.5 & - & - & - & - & 3.34 & - \\
\hline- & 0.53 & 0.56 & 0.56 & 0.57 & - & 0.54 & - & - & 0.57 & 0.6 & - \\
\hline- & - & - & - & $=$ & 4.17 & - & - & - & - & 4.32 & $\cdot$ \\
\hline- & - & - & - & : & 0.83 & - & - & - & - & 0.85 & - \\
\hline - & - & - & - & - & 2.48 & - & - & - & - & 2.59 & $\cdot$ \\
\hline - & 2.32 & 2.41 & 2.35 & 2.41 & 2.51 & 2.28 & - & - & 2.41 & 2.57 & - \\
\hline - & 0.35 & 0.36 & 0.36 & 0.36 & 0.43 & 0.36 & - & - & 0.36 & 0.43 & - \\
\hline - & 0.70372 & - & 0.70365 & 0.70365 & - & - & - & - & 0.70360 & - & - \\
\hline- & 0.512804 & - & 0.512801 & 0.512790 & - & - & - & - & 0.512769 & - & - \\
\hline - & 18.367 & - & 18.380 & 18.386 & - & - & - & - & 18.367 & - & - \\
\hline- & 15.507 & - & 15.511 & 15.510 & - & - & - & - & 15.500 & - & . \\
\hline - & 38.369 & - & 38.390 & 38.380 & - & - & - & - & 38.346 & - & - \\
\hline
\end{tabular}


Table 1 (continued).

\begin{tabular}{|c|c|c|c|c|c|c|c|c|c|c|c|}
\hline $802 \mathrm{~A}$ & $802 \mathrm{~A}$ & $802 \mathrm{~A}$ & $802 \mathrm{~A}$ & $802 \mathrm{~A}$ & $802 \mathrm{~A}$ & $802 \mathrm{~A}$ & $802 \mathrm{~A}$ & $802 \mathrm{~A}$ & $802 \mathrm{~A}$ & $802 \mathrm{~A}$ & $802 \mathrm{~A}$ \\
\hline $59 \mathrm{R}-1$ & $59 \mathrm{R}-1$ & 59R-1 & $59 \mathrm{R}-1$ & $59 \mathrm{R}-2$ & $59 \mathrm{R}-2$ & $59 \mathrm{R}-2$ & $59 R-2$ & $59 R-3$ & $59 \mathrm{R}-3$ & $59 \mathrm{R}-3$ & $59 R-3$ \\
\hline $70--75$ & $78--82$ & $130--136$ & $138-142$ & $8--13$ & $15-19$ & $100--105$ & $123--127$ & $17--22$ & $40--45$ & $86--90$ & $127--132$ \\
\hline 526.1 & 526.18 & 526.7 & 526.78 & 526.98 & 527.05 & 527.9 & 528.13 & 528.49 & 528.72 & 529.18 & 529.59 \\
\hline 8 & 8 & 9 & 9 & 9 & 9 & 10 & 11 & 12 & 13 & 13 & 13 \\
\hline & & & & & & & & & \multicolumn{3}{|c|}{ UM } \\
\hline 49.59 & 49.76 & 49.76 & 49.27 & 49.95 & 49.58 & 49.52 & 49.38 & 50.48 & 49.98 & 49.90 & 50.34 \\
\hline 1.22 & 1.14 & 1.10 & 1.10 & 1.24 & 1.11 & 1.21 & 1.10 & 1.16 & 1.23 & 1.01 & 1.20 \\
\hline 14.23 & 14.09 & 14.26 & 14.43 & 14.29 & 14.31 & 14.42 & 14.37 & 14.59 & 14.27 & 13.80 & 14.24 \\
\hline 2.41 & 2.73 & 3.55 & 3.99 & 2.97 & 3.30 & 2.60 & 3.65 & 3.18 & 2.03 & 2.74 & 2.65 \\
\hline 8.57 & 8.63 & 8.52 & 7.88 & 8.29 & 8.83 & 8.34 & 8.47 & 7.83 & 9.17 & 8.60 & 8.35 \\
\hline 0.20 & 0.20 & 0.21 & 0.21 & 0.19 & 0.21 & 0.20 & 0.21 & 0.18 & 0.21 & 0.21 & 0.21 \\
\hline 7.94 & 7.92 & 7.66 & 7.64 & 7.68 & 7.71 & 8.07 & 7.67 & 7.51 & 7.67 & 7.56 & 7.72 \\
\hline 12.13 & 12.02 & 11.85 & 11.58 & 11.85 & 12.02 & 12.12 & 11.85 & 11.84 & 11.85 & 12.00 & 11.78 \\
\hline 1.96 & 1.91 & 1.92 & 2.07 & 2.00 & 1.92 & 2.01 & 1.95 & 2.13 & 2.00 & 1.94 & 1.97 \\
\hline 0.06 & 0.08 & 0.14 & 0.18 & 0.20 & 0.14 & 0.05 & 0.13 & 0.11 & 0.10 & 0.12 & 0.08 \\
\hline 0.10 & 0.03 & 0.02 & 0.02 & 0.09 & 0.02 & 0.02 & 0.02 & 0.02 & 0.02 & 0.08 & 0.02 \\
\hline 1.14 & 1.04 & 1.03 & 1.09 & 1.05 & 0.90 & 0.99 & 0.91 & 0.87 & 0.84 & 1.08 & 1.08 \\
\hline 99.6 & 99.6 & 100.0 & 99.5 & 99.8 & 100.0 & 99.6 & 99.7 & 99.9 & 99.4 & 100.0 & 99.6 \\
\hline 0.26 & 0.18 & 0.07 & 0.24 & 0.13 & 0.13 & 0.27 & 0.07 & 0.24 & 0.18 & 0.11 & 0.10 \\
\hline \multirow[t]{2}{*}{0.43} & 0.53 & 0.92 & 0.73 & 0.58 & 0.54 & 0.50 & 0.71 & 0.51 & 0.50 & 0.60 & 0.86 \\
\hline & & & & & & & & & \multicolumn{3}{|c|}{ UM } \\
\hline 19 & 34 & 22 & 14 & 23 & 17. & 9 & 16 & 17 & 32 & 25 & 29 \\
\hline - & - & - & - & - & - & - & - & - & - & 57 & - \\
\hline 131 & 144 & 166 & 148 & 138 & 155 & 133 & 165 & 134 & 150 & 121 & 145 \\
\hline 164 & 155 & 152 & 152 & 157 & 151 & 157 & 151 & 151 & 150 & 167 & 156 \\
\hline 15 & 14 & 18 & 17 & 16 & 17 & 17 & 18 & 17 & 18 & - & 16 \\
\hline 5 & 3 & 4 & 4 & 5 & 4 & 4 & 5 & 4 & 4 & 24 & 4 \\
\hline 96 & 93 & 105 & 100 & 100 & 100 & 98 & 105 & 98 & 100 & 98 & 102 \\
\hline 1 & 4 & 4 & 5 & 4 & 6 & 6 & 5 & 6 & 4 & - & 6 \\
\hline 1 & 1 & 1 & 4 & 4 & 4 & 1 & 2 & 2 & 2 & $<10$ & 2 \\
\hline 244 & 249 & 245 & 255 & 249 & 263 & 246 & 259 & 407 & 266 & - & 264 \\
\hline 97 & 98 & 93 & 100 & 96 & 96 & 99 & 96 & 100 & 95 & 78 & 95 \\
\hline 336 & 344 & 345 & 350 & 349 & 338 & 340 & 349 & 346 & 348 & - & 346 \\
\hline 25 & 25 & 25 & 25 & 25 & 25 & 25 & 25 & 26 & 25 & 16 & 25 \\
\hline 93 & 88 & 96 & 100 & 94 & 95 & 90 & 97 & 92 & 95 & 92 & 93 \\
\hline 64 & 65 & 64 & 65 & 0 & 65 & 64 & 64 & 64 & 63 & 62 & 63 \\
\hline \multicolumn{4}{|c|}{ WU } & \multicolumn{6}{|c|}{ WU } & \multicolumn{2}{|r|}{ WU } \\
\hline- & - & 50 & - & - & - & - & 48 & - & - & - & 50 \\
\hline- & - & 0.08 & - & - & - & 0.10 & 0.16 & - & - & - & 0.20 \\
\hline- & - & 1.65 & - & - & - & 1.60 & 1.69 & - & - & - & 1.76 \\
\hline - & - & 48.10 & - & - & - & 47.60 & 48.90 & - & - & - & 48.50 \\
\hline - & - & 0.18 & - & - & - & 0.17 & 0.19 & - & - & - & 0.17 \\
\hline- & - & 0.22 & - & - & - & 0.26 & 0.20 & - & - & - & 0.20 \\
\hline - & $\cdot$ & 0.30 & - & - & - & 0.07 & 0.40 & - & - & - & 0.30 \\
\hline - & - & 3.16 & - & - & . & 4.86 & 3.14 & - & - & - & 3.13 \\
\hline- & - & 8.10 & - & - & - & 10.82 & 8.70 & - & - & - & 8.30 \\
\hline- & - & - & - & - & - & 1.51 & - & - & - & - & - \\
\hline - & - & 5.00 & - & - & - & 7.94 & 8.00 & - & - & - & 17.00 \\
\hline - & - & 2.35 & - & - & - & 2.20 & 2.31 & - & - & - & 2.25 \\
\hline- & - & 0.86 & - & - & - & 0.88 & 0.88 & - & - & - & 0.86 \\
\hline - & $\cdot$ & - & - & - & - & 3.15 & - & - & - & - & - \\
\hline - & - & 0.57 & - & - & - & - & 0.56 & - & - & - & 0.60 \\
\hline - & - & - & - & - & - & 4.05 & - & - & - & - & - \\
\hline - & - & - & - & - & - & 0.80 & - & - & - & - & - \\
\hline - & - & - & - & - & - & 2.43 & - & - & - & - & - \\
\hline - & - & 2.37 & - & - & - & 2.47 & 2.43 & - & - & - & 2.36 \\
\hline - & - & 0.36 & - & - & - & 0.42 & 0.36 & - & - & - & 0.37 \\
\hline- & - & - & - & - & - & - & 0.70361 & - & - & - & - \\
\hline- & - & - & - & - & - & - & 0.512789 & - & - & - & - \\
\hline - & - & - & - & - & - & - & 18.380 & - & - & - & - \\
\hline - & - & - & - & - & - & - & 15.491 & - & - & - & - \\
\hline - & - & - & - & - & - & - & 38.336 & - & - & - & - \\
\hline
\end{tabular}


Table 1 (continued).

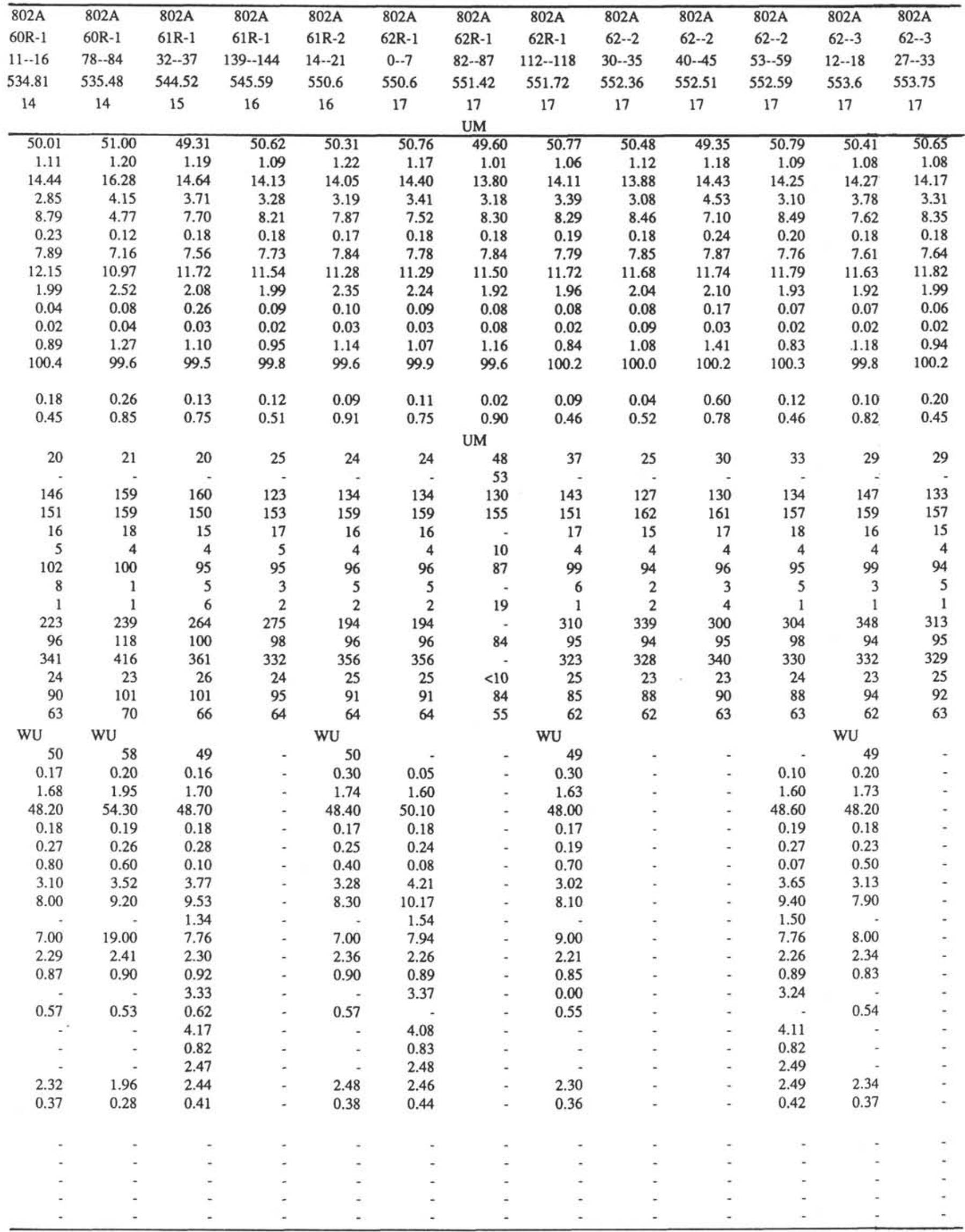


Table 2. Comparison of interlaboratory results for the duplicate analysis of selected Leg 129 basalts at University of Keele (UK) and Washington University (WU).

\begin{tabular}{|c|c|c|c|c|c|c|c|c|}
\hline \multirow{2}{*}{$\begin{array}{l}\text { Hole: } \\
\text { Core, section: } \\
\text { Interval }(\mathrm{cm}) \text { : } \\
\text { Analytical laboratory: }\end{array}$} & \multicolumn{2}{|c|}{$\begin{array}{c}802 \mathrm{~A} \\
59 \mathrm{R}-1 \\
7-12\end{array}$} & \multicolumn{2}{|c|}{$\begin{array}{l}802 \mathrm{~A} \\
61 \mathrm{R}-1 \\
32-37\end{array}$} & \multicolumn{2}{|c|}{$\begin{array}{c}801 \mathrm{C} \\
2 \mathrm{R}-1 \\
112-118\end{array}$} & \multicolumn{2}{|c|}{$\begin{array}{l}801 \mathrm{C} \\
10 \mathrm{R}-6 \\
67-70\end{array}$} \\
\hline & UK & WU & UK & WU & UK & WU & UK & WU \\
\hline \multicolumn{9}{|l|}{ Major oxides (wt\%) } \\
\hline $\mathrm{FeO}^{*}$ & 10.87 & 11.11 & 11.04 & 11.31 & 9.15 & 8.79 & 9.70 & 10.04 \\
\hline $\mathrm{CaO}$ & 11.97 & 11.80 & 11.72 & 11.70 & 7.14 & 6.70 & 12.26 & 11.50 \\
\hline $\mathrm{Na}_{2} \mathrm{O}$ & 2.08 & 2.06 & 2.08 & 2.07 & 3.94 & 3.53 & 2.77 & 2.74 \\
\hline \multicolumn{9}{|l|}{ Trace element (ppm) } \\
\hline $\mathrm{Ba}$ & 7 & b.d. & 20 & b.d. & 396 & 341 & 9 & b.d \\
\hline $\mathrm{Cr}$ & 139 & 153 & 160 & 155 & 87 & 92.1 & 200 & 198 \\
\hline Cs & 0.10 & 0.11 & 0.16 & 0.24 & 0.44 & 0.39 & 0.10 & b.d. \\
\hline Hf & 1.50 & 1.75 & 1.70 & 1.74 & 5.7 & 5.9 & 2.60 & 2.63 \\
\hline $\mathrm{Ni}$ & 103 & 107 & 95 & b.d. & 42 & 43 & 67 & 70 \\
\hline $\mathrm{Rb}$ & 1 & b.d. & 6 & b.d. & 44 & 43 & 1 & b.d. \\
\hline Sc & 47.6 & 50.2 & 48.7 & 50.1 & 21.5 & 20.4 & 45.9 & 48.4 \\
\hline $\mathrm{Sr}$ & 100 & 104 & 100 & 100 & 477 & 500 & 118 & 80 \\
\hline $\mathrm{Ta}$ & 0.17 & 0.19 & 0.18 & 0.18 & 2.46 & 2.63 & 0.124 & 0.123 \\
\hline Th & 0.24 & 0.27 & 0.28 & 0.18 & 2.43 & 2.43 & 0.109 & b.d. \\
\hline $\mathrm{U}$ & 0.11 & b.d. & 0.097 & b.d. & 0.81 & 0.96 & 0.10 & b.d. \\
\hline $\mathrm{Zr}$ & 66 & 60 & 66 & b.d. & 259 & 230 & 101 & 120 \\
\hline \multicolumn{9}{|c|}{ Rare earth elements (ppm) } \\
\hline $\mathrm{La}$ & 3.96 & 3.23 & 3.77 & 3.41 & 31.16 & 27.50 & 3.26 & 2.80 \\
\hline $\mathrm{Ce}$ & 10.28 & 8.70 & 9.53 & 8.90 & 64.62 & 58.60 & 10.08 & 9.20 \\
\hline Sm & 2.35 & 2.41 & 2.30 & 2.42 & 6.93 & 6.93 & 3.33 & 3.47 \\
\hline Eu & 0.91 & 0.91 & 0.92 & 0.92 & 2.32 & 2.30 & 1.30 & 1.32 \\
\hline $\mathrm{Yb}$ & 2.57 & 2.51 & 2.44 & 2.41 & 2.58 & 2.47 & 3.68 & 3.62 \\
\hline $\mathrm{Lu}$ & 0.43 & 0.378 & 0.41 & 0.386 & 0.42 & 0.345 & 0.62 & 0.53 \\
\hline
\end{tabular}

Note: b.d. = beyond detection limit. 